\title{
The Shape of Orthogonal Cycles in Three Dimensions
}

\author{
Giuseppe Di Battista • Ethan Kim • \\ Giuseppe Liotta · Anna Lubiw • Sue Whitesides
}

Received: 6 December 2010 / Revised: 14 October 2011 / Accepted: 16 October 2011 /

Published online: 8 November 2011

(C) Springer Science+Business Media, LLC 2011

\begin{abstract}
Let $\sigma$ be a directed cycle whose edges have each been assigned a desired direction in $3 D$ (East, West, North, South, Up, or Down) but no length. We say that $\sigma$ is a shape cycle. We consider the following problem. Does there exist an orthogonal representation $\Gamma$ of $\sigma$ in $3 D$ space such that no two edges of $\Gamma$ intersect except at common endpoints and such that each edge of $\Gamma$ has the direction specified in $\sigma$ ? If the answer is positive, we say that $\sigma$ is simple. This problem arises in the context of extending orthogonal graph drawing techniques from $2 D$ to $3 D$. We give a combinatorial characterization of simple shape cycles that yields linear time recognition and drawing algorithms.
\end{abstract}

Keywords Graph drawing - 3D orthogonal drawing - Topology-shape-metrics · Cycles

Communicated by János Pach.

A preliminary (and incomplete) version of this paper appeared as [3].

G. Di Battista $(\bowtie)$

Dipartimento di Informatica ed Automazione, Università di Roma Tre, Roma, Italy

e-mail: gdb@dia.uniroma3.it

E. Kim

School of Computer Science, McGill University, Montreal, Canada

e-mail: ethan@cs.mcgill.ca

G. Liotta

Dipartimento di Ingegneria Elettronica e dell'Informazione, Università di Perugia, Perugia, Italy e-mail: liotta@diei.unipg.it

\author{
A. Lubiw \\ Department of Computer Science, University of Waterloo, Waterloo, ON, Canada \\ e-mail: alubiw@uwaterloo.ca \\ S. Whitesides \\ Department of Computer Science, University of Victoria, Victoria, BC, Canada \\ e-mail: sue@uvic.ca
}




\section{Introduction}

Consider a cycle with an orthogonal drawing in $3 D$, where each edge is represented by a line segment parallel to one of the coordinate axes. Traversing the cycle, we obtain a cyclic sequence of directions East, West, North, South, Up, or Down, one per edge. Such a cyclic sequence of directions is called a shape cycle. In this paper we characterize the shape cycles that arise from simple orthogonal cycles in $3 D$, where a cycle is simple if no two edges intersect except at common endpoints.

We first elaborate on the problem statement. A shape cycle $\sigma$ is specified by a cyclic sequence of elements, each assigned a direction label from the set $\{E, W, N, S, U, D\}$. The simplicity testing problem for $\sigma$ is to decide whether there exists an orthogonal drawing $\Gamma(\sigma)$ of cycle $\sigma$ so that $\Gamma(\sigma)$ is simple and has the sequence of direction labels $\sigma$. If the shape cycle $\sigma$ admits such a drawing, then $\sigma$ is said to be simple.

Not all shape cycles are simple. For example, the shape cycle $\sigma=E S U N D W U N$ shown in Fig. 1 has no simple orthogonal drawing, even though each direction label appears at least once. To see this, note that if we try to avoid the edge crossing by making $e_{4}$ longer, we end up in the North half-space, with no hope of return. For a positive example, observe that changing the final direction of $\sigma$ from $N$ to $S$ yields a simple shape cycle.

Our main result is a combinatorial characterization of simple shape cycles, which yields linear time testing and drawing algorithms. The characterization is relatively easy to state, but neither sufficiency nor necessity is obvious. In $2 D$, simple shape cycles were characterized by Vijayan and Widgerson [10] in 1985. The situation in $3 D$ is substantially more difficult, which is perhaps not surprising given that simple cycles in $3 D$ are knots. We motivate our characterization by comparing with the $2 D$ case.

Vijayan and Widgerson [10] proved that a $2 D$ shape cycle is simple if and only if it contains either four more right turns than left turns, or four more left turns than right turns. The forward direction (necessity) is proved by summing the interior angles of the polygon. This approach is not possible in $3 D$-in particular, the concepts of

Fig. 1 A non-simple drawing of shape cycle $\sigma=e_{1} e_{2} \ldots e_{8}=E S U N D W U N$. Edges $e_{2}, e_{3}, e_{4}$, and $e_{5}$ form a flat of $\sigma$

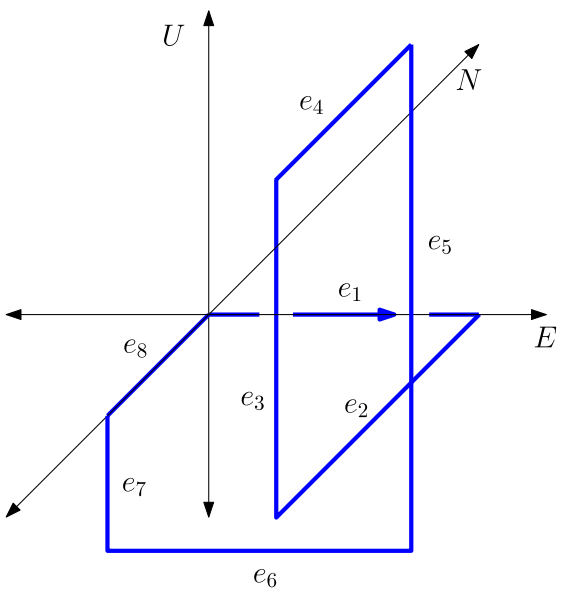


turns and interior angles are not applicable because, while a cycle drawn in $2 D$ is a rectilinear polygon bounding an area, a $3 D$ cycle does not bound a volume or a disc, and in fact may form any knot. However, the proof of sufficiency in [10] is relevant to the $3 D$ situation. Vijayan and Widgerson perform reductions that eliminate pairs of elements from a shape cycle. For example $E N E$ is reduced to $E$, eliminating one left and one right turn. These reductions are performed until all that remains is a "skeleton" sequence of the four distinct labels $\{N, S, E, W\}$, with consecutive labels orthogonal. This skeleton is drawn as a rectangle, and, reversing the reductions, the drawing is elaborated by adding back the eliminated pairs. Our characterization of $3 D$ simple shape cycles has a similar flavor: we prove that a $3 D$ shape cycle is simple if and only if it has an (appropriately defined) "skeleton" of six elements, one of each direction label. Although we are unable to find the $3 D$ skeleton using reductions, our proof of sufficiency is similar to the $2 D$ case in that we draw the 6-element skeleton along the edges of a cube and then elaborate the drawing to add the extra edges.

We state our characterization in Sect. 2. The characterization is interesting in its own right and also has application to graph drawing via the topology-shape-metrics approach, as discussed next.

The topology-shape-metrics approach [2] for constructing orthogonal drawings of planar graphs in $2 D$ is a widely studied Graph Drawing technique. It consists of three steps, called planarization, orthogonalization, and compaction. The planarization step determines a planar topology of the drawing, i.e., for each vertex, it chooses the circular ordering of its incident edges such that the resulting drawing is planar. The orthogonalization step determines the shape of the drawing, i.e., each edge is equipped with a sequence of labels that define the shape it will have in the final drawing. For example, an edge $(u, v)$ could be labeled $N E S N E$, meaning "starting from $u$ first go North, then go East, etc." Notice that the labels of the orthogonalization step have exactly the same meaning introduced above in this paper. Finally, the compaction step assigns coordinates to vertices and bends without introducing crossings and while preserving the previously specified shapes of the edges.

An essential prerequisite for the feasibility of the topology-shape-metrics approach is that the orthogonalization step produces labels that the compaction step can transform into segments without introducing crossings. Hence, the relationship between orthogonalization and compaction has been studied in several papers, including $[1,5,8,10,11]$.

Although the literature on $3 D$ Graph Drawing is quite rich-see, for example, the survey on this topic by Dujmović and Whitesides [9]—nobody has been able to extend the topology-shape-metrics approach to $3 D$, even for limited classes of graphs. One of the key reasons is the result of Patrignani [7] who proved that it is NP-hard to decide if a degree-six graph with direction constraints on its edges has an orthogonal $3 D$ drawing without edge intersections. By providing a linear time recognition algorithm for simple shape cycles, this paper makes a step towards understanding to what extent the topology-shape-metrics approach can be scaled to $3 \mathrm{D}$.

In the next section we state our combinatorial characterization for simple shape cycles, give an overview of our method, and describe the organization of the rest of the paper. 
Fig. 2 Shape cycle $\sigma=E S U N D W U N$ and its four flats, $F_{1}, \ldots, F_{4}$

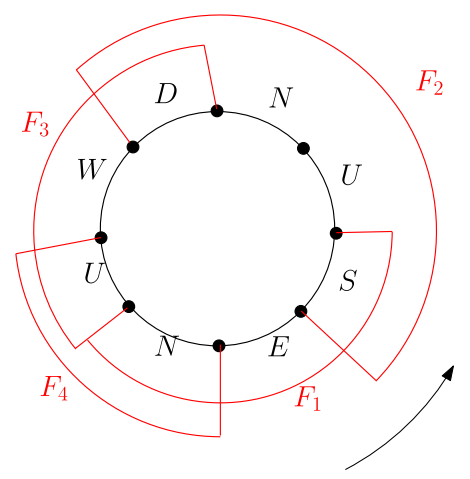

\section{Overview}

Our characterization of simple shape cycles depends on two fundamental concepts which we motivate as follows. A $3 D$ (non-planar) shape cycle must certainly include all six directions in order to close up, but that is not sufficient for the shape cycle to be simple, as shown by the example in Fig. 1. One's first intuition is that simplicity should usually be possible in $3 D$-even though it is relatively uncommon in $2 D$ because space is big and edges are thin. However, as Fig. 1 shows, the condition of simplicity in a plane can affect the whole drawing. In this example the subsequence $e_{2} e_{3} e_{4} e_{5}=S U N D$ lies in one plane, and because of simplicity, the $S$ and $D$ edges cannot both be the right length to close up the cycle. Such a blockage in one plane will not always make $\sigma$ non-simple, but in this example it does, because $S$ and $D$ do not occur elsewhere in $\sigma$. We now formalize these concepts.

An isothetic plane is a plane orthogonal to one of the coordinate axes.

Definition 1 A flat $F$ of a shape path or cycle $\sigma$ is a maximal consecutive subsequence of $\sigma$ such that every element in $F$ has a direction label parallel to the same isothetic plane.

For example, consider the shape cycle $\sigma=e_{1} e_{2} \ldots e_{8}=E S U N D W U N$ of Fig. 1. In Fig. 2, we show the four flats of $\sigma$, namely $F_{1}=e_{8} e_{1} e_{2}=N E S, F_{2}=e_{2} e_{3} e_{4} e_{5}=$ $S U N D, F_{3}=e_{5} e_{6} e_{7}=D W U$, and $F_{4}=e_{7} e_{8}=U N$.

Definition 2 A not necessarily consecutive subsequence $\tau$ of $\sigma$ is a canonical sequence if: (1) the direction labels of the elements of $\tau$ are distinct; (2) each flat of $\sigma$ contains at most three elements of $\tau$; and (3) if a flat $F$ of $\sigma$ contains more than one element of $\tau$, then $\tau \cap F$ forms a consecutive subsequence of $\sigma$. A canonical sequence is a full canonical sequence if it contains all six direction labels.

For example, in the shape cycle $\sigma=E S U N D W U N$ of Fig. 1, each of $e_{1} e_{2} e_{3}$ and $e_{1} e_{3}$ is a canonical sequence for $\sigma$. However, $e_{2} e_{3} e_{4} e_{5}$ is not a canonical sequence because all four elements belong to the same flat. Observe that $\sigma$ does not contain a full canonical sequence. Such a full canonical sequence $\tau$ would need to contain $e_{2}=S$ 
and $e_{4}=D$ because there are no other occurrences of $S$ or $D$ in $\sigma$. By condition (3), $\tau$ must also contain $e_{2}$ and $e_{3}$. But then this violates condition (2).

Now we state our main result.

Theorem 1 A $3 D$ shape cycle is simple if and only if it contains a full canonical sequence.

The proof of Theorem 1 uses our previous work [4], where we introduced a simpler notion of canonical sequence in the context of solving the shape path reachability problem. The shape path reachability problem is to determine, given a shape path $\sigma$ and a point $p$ in the interior of an octant, whether $\sigma$ admits a simple drawing that starts at the origin, ends at $p$, and respects the direction labels for its edges. The main result of [4] can be summarized in concrete terms for a particular octant in $3 D$ as follows.

Theorem 2 (3D Path [4]) Let $p$ be a point in the UNE octant. Then a shape path $\sigma$ admits a simple orthogonal drawing that starts at the origin and terminates at $p$ if and only if $\sigma$ contains a canonical sequence with direction labels $\{U, N, E\}$.

If a $3 D$ shape cycle $\sigma$ admits a simple orthogonal drawing, then the drawing of $\sigma$ can be regarded as a concatenation of two shape paths traveling to opposite octants. Therefore, one may hope to find a full canonical sequence by first partitioning the shape cycle into two shape paths $\sigma_{1}$ and $\sigma_{2}$ traveling between opposite octants, and then finding the canonical sequence of length three for each subpath via Theorem 2 (3D Path), and finally, concatenating the two canonical sequences to obtain a full canonical sequence. The difficulty with this approach is that the final concatenation does not necessarily yield a canonical sequence. There may be "bridging" flats that overlap both $\sigma_{1}$ and $\sigma_{2}$ at the two places where they join. If elements from the two separate canonical sequences for $\sigma_{1}$ and $\sigma_{2}$ appear in such a bridging flat, they may violate the conditions for a canonical sequence. In particular, they may fail to be consecutive in the bridging flat, and moreover, there may be four of them in one bridging flat. Thus the necessity of the condition of Theorem 1 is not an immediate consequence of Theorem 2 ( $3 D$ Path). Although we will indeed follow this approach, we must be more careful about how we split the cycle into paths, and we must do more work to merge the canonical sequences.

The rest of this paper is organized as follows. Further definitions and preliminary results are in Sect. 3. Section 4 gives the proof of necessity of our condition, i.e. the proof that a simple $3 D$ shape cycle contains a full canonical sequence. Sufficiency is proved in Sect. 5; the proof is constructive and provides an algorithm to construct a simple drawing of the shape cycle. Section 6 gives a linear time algorithm for testing the simplicity of a shape cycle. Conclusions are in Sect. 7.

\section{Preliminaries}

This section contains further definitions and properties that are used throughout the paper. 
Formally, a shape cycle is a (graph theoretic) directed cycle where each edge has a direction label from the set $\{E, W, N, S, U, D\}$. Similarly, a shape path is a directed path where each edge has a direction label. Thus the elements of a shape path or cycle are its edges, and we will use the terms "elements" and "edges" interchangeably. We will sometimes write a shape path or cycle $\sigma$ as a sequence of elements $e_{1} e_{2} \ldots e_{n}$. For an element $e$ of $\sigma$, we will write $e=a b$ to indicate that edge $e$ is a directed edge from vertex $a$ to vertex $b$.

Recall that a flat is defined as a subsequence of the elements of a path shape or cycle-see Definition 1. We extend this to vertices in the obvious way: a vertex $v$ of shape path or cycle $\sigma$ is contained in a flat $F$ of $\sigma$ if $F$ contains an element with $v$ as its endpoint. For two vertices $u$ and $v$ in a shape path or cycle $\sigma$, the portion of $\sigma$ from $u$ to $v$ is denoted by $\sigma_{u v}$. If all the elements in a flat $F$ are encountered when traversing from vertex $u$ to vertex $v$, we say that $\sigma_{u v}$ contains the flat $F$.

We shall only consider shape paths or cycles such that every two consecutive elements are orthogonal. Thus we forbid, e.g. the consecutive pairs $N N$ and $N S$.

We assume that any $3 D$ shape path or cycle consists of at least two flats, because otherwise it lies in $2 D$. A flat is said to be light if it contains exactly two elements and heavy otherwise. Every two consecutive flats share one element, which we call a transition element. A transition element lies in exactly two flats, and any other element lies in exactly one flat. In any drawing, two consecutive flats must be drawn in two orthogonal planes, and the shared transition element lies on the line of intersection of the two planes. Observe that in a shape cycle, every flat begins and ends with a transition element. A transition vertex is the middle vertex of a light flat. Thus there is a transition vertex whenever two transition edges are incident. For example, the shape cycle shown in Figs. 1 and 2 has four flats and thus has four transition elements; it has one light flat and thus has one transition vertex.

It is easier to understand shape cycles and canonical sequences when they are written as sequences of direction labels. For example, $\sigma=N E N U W D S E D$ with canonical sequence $\tau=N D S$ rather than $\sigma=e_{1} e_{2} \ldots e_{9}$ with $d\left(e_{1}\right)=N$, etc., and $\tau=e_{1} e_{6} e_{7}$. However, the former notation does not distinguish which of the elements labeled $N$ is in $\tau$. To make such distinctions, we will denote elements by accented direction labels. For example, $\sigma=\bar{N} \bar{E} \hat{N} \bar{U} \bar{W} \bar{D} \bar{S} \hat{E} \hat{D}$ contains the canonical sequence $\tau=\bar{N} \bar{D} \bar{S}$ and the canonical sequence $\tau^{\prime}=\hat{N} \bar{D} \bar{S}$.

We will have occasion to refer to the elements of a canonical sequence $\tau$ as a set, without specifying their order (which is completely determined from $\sigma$ anyway). Thus we will say that $\tau=\{\bar{N}, \bar{S}, \bar{E}, \bar{D}\}$ is a canonical sequence in $\sigma$. This allows us to combine canonical sequences; for example if $\tau_{1}=\{\bar{U}, \bar{N}, \bar{E}\}$ and $\tau_{2}=\{\hat{N}, \hat{D}, \bar{E}\}$, then $\tau_{1} \cap \tau_{2}=\{\bar{E}\}$ and $\tau_{1} \cup \tau_{2}=\{\bar{U}, \bar{N}, \bar{E}, \hat{N}, \hat{D}\}$. (Note that the result need not be a canonical sequence.) Sometimes we care only about some elements of a canonical sequence. If $\tau$ is a canonical sequence that contains a subset $A$ of elements, we write $\tau=A^{+}$. For example, we would write $\tau=\{\bar{N}, \bar{E}\}^{+}$for a canonical sequence that contains $\bar{N}, \bar{E}$, and possibly some other elements.

Finally, the type of a canonical sequence is just the set of direction labels contained in $\tau$. For example, we might say that $\tau$ is a canonical sequence of type $\{U, N, E, S\}$ or of type $\{U, N, S\}^{+}$without specifying which elements of $\sigma$ are in $\tau$.

We now make a note about symmetries. Many of our results will be stated for only one choice of directions (for example, Theorem 2 (3D Path) was stated for the $U N E$ 
octant) but will be applied for other directions, as justified by the following. Let $\varphi$ be a permutation of the six direction labels that maps opposite pairs of labels to opposite pairs of labels (for example, $\varphi$ might map $N, S, E, W, U, D$ to $E, W, N, S, D, U$, respectively). Then $\varphi$ defines a linear transformation of $3 D$ space that determines a bijection between simple drawings of $\sigma$ and simple drawings of $\varphi(\sigma)$, and therefore a result that holds for $\sigma$ also holds for $\varphi(\sigma)$.

Another symmetry we will make use of is the possibility of traversing a shape cycle in the negative sense, which means reversing the sequence and exchanging $N$ and $S, E$ and $W$, and $U$ and $D$.

Relative to a placement of the origin at some point $p$, we refer to, e.g. the $E$ axis, the $N E$ quadrant, and the $U N E$ octant. All of these are open sets, so $3 D$ space is a disjoint union of the 8 octants, 12 quadrants, 6 axes, and the origin $p$. We say that two points $u$ and $v$ are in an octant relation if they are not contained in a common isothetic plane. In Sect. 4.2 we will extend this definition to edges and also define a quadrant relation.

\section{Necessity}

In this section, we prove that if a $3 D$ shape cycle $\sigma$ admits a simple drawing $\Gamma(\sigma)$, then it has a full canonical sequence.

As explained in the Overview (Sect. 2), the basic plan is to take two vertices of $\Gamma(\sigma)$ that lie in opposite octants, say the $U N E$ and $D S W$ octants, and cut $\sigma$ at those vertices to obtain two paths. By Theorem 2 (3D Path), the paths have canonical sequences of types $\{U, N, E\}$ and $\{D, S, W\}$, and we hope to concatenate them to form a full canonical sequence for the whole cycle $\sigma$. The trouble is that the concatenated sequence may fail to be canonical because a "bridging" flat that overlaps both paths may contain non-consecutive canonical elements or more than three canonical elements. This problem is avoided if the bridging flats are light, i.e. if we break $\sigma$ at transition vertices.

Therefore, if a simple shape cycle has two transition vertices in an octant relation, then it has a full canonical sequence.

Unfortunately, this situation is far from general. First of all, a shape cycle may have no transition vertices at all. We may need to break $\sigma$ into two paths in more general ways, by removing transition edges, or even by allowing paths that overlap on a transition edge. This makes the arguments for concatenating or "merging" canonical sequences more complicated. Secondly, breaks of the type that work for merging of canonical sequences cannot be guaranteed to lie in opposite octants. We will therefore need results that guarantee canonical sequences in paths that are more general than paths going between opposite octants. For example, one of our main lemmas (the "3D U-Turn" Lemma) is about canonical sequences of length four in a simple path drawn in such a way that it leaves an isothetic plane and then returns to the plane. As the name suggests, the canonical sequence will contain a pair of opposite direction labels.

This section is organized as follows. Section 4.1 gathers together the various results we will need for our proof of necessity: Section 4.1.1 discusses a general position assumption for shape cycles; Section 4.1.2 has results about merging canonical 
sequences from two (almost) disjoint subpaths of a shape cycle; and Sect. 4.1.3 is about canonical sequences in simple shape paths of various kinds, and includes the “3D U-Turn” Lemma. Finally, the proof of necessity is in Sect. 4.2.

\subsection{Tools for Proof of Necessity}

As described above, this section contains results that will be used in our proof of necessity.

\subsubsection{General Position}

In this section we define a general position property for shape cycles and show that we can assume general position without loss of generality. In any drawing $\Gamma(\sigma)$, every flat $F$ of $\sigma$ lies in an isothetic plane $\mathcal{P}$. It may happen that $\mathcal{P}$ is crossed by other edges of the drawing, but we would like to avoid the situation where vertices outside $F$ lie in $\mathcal{P}$. More generally:

Definition 3 A simple drawing $\Gamma(\sigma)$ of a shape path or cycle $\sigma$ is in general position if, for every isothetic plane $\mathcal{P}$, the set of vertices of $\sigma$ lying in $\mathcal{P}$ forms a path in $\sigma$.

Note the consequence that the set of vertices lying in $\mathcal{P}$ is contained in a flat of $\sigma$ and in fact will form a flat unless the set has just two vertices. A further consequence is that two vertices are in an octant relation if and only if they do not share a flat. Therefore, being in an octant relation becomes a property of the shape cycle rather than of its drawing.

Lemma 1 A simple drawing $\Gamma(\sigma)$ of a shape path or cycle $\sigma$ can always be perturbed to place it in general position.

Proof The proof is by induction on the number of isothetic planes violating the general position condition. Let $\mathcal{P}$ be such a plane. Let $\sigma_{1}, \sigma_{2}, \ldots, \sigma_{t}$ with $t>1$ be the connected paths of $\sigma$ lying in $\mathcal{P}$. We will stretch $\Gamma(\sigma)$ to put each $\sigma_{i}$ on its own plane. Assume without loss of generality that $\mathcal{P}$ is an $N S U D$ plane. Let $S_{E}$ be the vertices that lie East of $\mathcal{P}$. All the vertices of $S_{E}$ are translated East $t$ units. For $i=1, \ldots, t$, let $S_{i}$ be the set of vertices of $\sigma_{i}$. All the vertices of $S_{i}$ are translated East $i-1$ units.

Note that the drawing remains simple. To see that it remains orthogonal, consider an edge $u v$. If at least one of $u, v$ lies outside $\mathcal{P}$, then either the two vertices are translated the same distance, or the edge $u v$ is perpendicular to a NSUD plane, and in either case the stretch operation leaves $u v$ orthogonal. On the other hand, if $u$ and $v$ both lie in $\mathcal{P}$, then, because they are joined by an edge, they lie in the same set $S_{i}$ and are translated the same distance.

Observe that the plane $\mathcal{P}$ that violated the general position assumption has been replaced by $t$ planes that each satisfy the general position assumption. Other isothetic planes that contain vertices are the same before and after. Therefore the proof follows by induction. 
As a result of Lemma 1, we shall assume henceforth that all drawings are in general position. This has the following consequence regarding vertices that lie on an isothetic line.

Lemma 2 (General position) Let $\Gamma(\sigma)$ be a simple drawing of a shape path or cycle $\sigma$, and let $u$ and $v$ be two vertices that lie on an isothetic line in $\Gamma(\sigma)$. Then either $u v$ is an edge, or $\sigma$ is a shape cycle that splits into $\sigma_{u v}$ and $\sigma_{v u}$, two flats that lie in orthogonal planes in $\Gamma(\sigma)$.

Proof Suppose that $u v$ is not an edge. Vertices $u$ and $v$ lie on two orthogonal isothetic planes. By the general position assumption, there is a path in each plane containing $u$ and $v$. Each path forms a flat. (Note that each of $u$ [and $v$ ] is either a transition vertex, in which case there is an additional light flat centered at it, or is incident to a transition edge that lies in the line of intersection of the two planes.)

\subsubsection{Merging Lemmas}

This section is about conditions for merging canonical sequences of subpaths of a shape cycle. We must avoid the situation where some flat "bridges" the two subpaths and contains canonical elements on each side thereby acquiring more than three canonical elements or non-consecutive canonical elements.

We say that two subpaths or canonical sequences of a shape path or cycle are disjoint if they do not have elements in common; they may share a vertex, and the same direction label may appear in both of them. We need two more specialized notions of disjointness:

Definition 4 Let $\tau_{1}$ and $\tau_{2}$ be two canonical sequences of a shape path or cycle. Then $\tau_{1}$ and $\tau_{2}$ are direction-disjoint if $\tau_{1}$ and $\tau_{2}$ do not share a direction label.

Definition 5 Let $\sigma_{1}$ and $\sigma_{2}$ be subpaths of a shape path or cycle $\sigma$. Then $\sigma_{1}$ and $\sigma_{2}$ are flat-separated if they are disjoint and no heavy flat contains an element of $\sigma_{1}$ and an element of $\sigma_{2}$.

Observe that the property of being flat-separated is equivalent to the property that between $\sigma_{1}$ and $\sigma_{2}$ (and between $\sigma_{2}$ and $\sigma_{1}$ in the case of a shape cycle), either there is a shared transition vertex, or there is a transition edge (or more).

Our most basic merging lemma is as follows.

Lemma 3 (Basic merge) Let $\sigma$ be a shape path or cycle with flat-separated subpaths $\sigma_{1}$ and $\sigma_{2}$. Let $\tau_{1}$ and $\tau_{2}$ be direction disjoint canonical sequences for $\sigma_{1}$ and for $\sigma_{2}$, respectively. Then $\tau=\tau_{1} \cup \tau_{2}$ is a canonical sequence for $\sigma$.

Proof We verify the conditions for $\tau$ to be a canonical sequence. The direction labels of the elements of $\tau$ are distinct because $\tau_{1}$ and $\tau_{2}$ are direction-disjoint canonical sequences. Because $\sigma_{1}$ and $\sigma_{2}$ are flat-separated, a flat $F$ of $\sigma$ is either a light flat or is disjoint from $\sigma_{1}$ or disjoint from $\sigma_{2}$. In all cases, $F \cap \tau$ contains at most three elements, and they must be consecutive in $\sigma$. 
In some situations, we want to merge canonical sequences that are not directiondisjoint. We therefore need to be able to remove elements from canonical sequences. We begin with a condition that allows such removal.

Definition 6 A canonical sequence $\tau$ is orthogonal if any two elements of $\tau$ have orthogonal direction labels (i.e. $\tau$ does not contain two elements with opposite direction labels).

Observe that if $\tau$ is an orthogonal canonical sequence, then deleting elements of $\tau$ leaves an orthogonal canonical sequence. This is because no flat can contain three elements of $\tau$.

Lemma 4 (Merge) Let $\sigma$ be a shape path or cycle with flat-separated subpaths $\sigma_{1}$ and $\sigma_{2}$. Let $\tau_{1}$ and $\tau_{2}$ be canonical sequences for $\sigma_{1}$ and for $\sigma_{2}$, respectively, such that at least one of $\tau_{1}$ or $\tau_{2}$ is orthogonal. Then $\sigma$ has a canonical sequence $\tau$ whose set of direction labels is equal to the union of the direction labels in $\tau_{1}$ and the direction labels in $\tau_{2}$.

Proof Assume without loss of generality that $\tau_{1}$ is orthogonal. Create $\tau_{1}^{\prime}$ by removing from $\tau_{1}$ any element whose direction label also appears in $\tau_{2}$. Then $\tau_{1}^{\prime}$ is a canonical sequence for $\sigma_{1}$, and $\tau_{1}^{\prime}$ and $\tau_{2}$ are direction-disjoint. By Lemma 3 (Basic Merge), $\tau_{1}^{\prime} \cup \tau_{2}$ is a canonical sequence for $\sigma$. Note that the set of direction labels of $\tau_{1}^{\prime} \cup \tau_{2}$ is the union of those of $\tau_{1}$ and of $\tau_{2}$.

Finally, there will be some situations where we will be unable to find flat-separated subpaths and will need a more complicated merging condition for the case in which the two subpaths share a transition element.

Lemma 5 (Overlap merge) Let $\sigma$ be a shape path or cycle, and let e be a transition element of $\sigma$. Let $\sigma_{1}$ and $\sigma_{2}$ be two subpaths of $\sigma$ that share exactly e. Let $\tau_{1}$ and $\tau_{2}$ be canonical sequences for $\sigma_{1}$ and for $\sigma_{2}$, respectively, such that:

(1) $\sigma_{1}-\{e\}$ and $\sigma_{2}-\{e\}$ are flat-separated. (Note that this condition is vacuous for paths.)

(2) The direction label of e appears in both $\tau_{1}$ and $\tau_{2}$.

(3) At least one of $\tau_{1}$ and $\tau_{2}$ is orthogonal.

Then, $\sigma$ has a canonical sequence $\tau$ whose set of direction labels is equal to the union of the direction labels in $\tau_{1}$ and the direction labels in $\tau_{2}$.

Proof Assume without loss of generality that $\tau_{1}$ is orthogonal. We will construct $\tau$ by removing elements from $\tau_{1} \cup \tau_{2}$ to eliminate duplicate direction labels. If $e \in \tau_{1} \cup \tau_{2}$ and another element in $\tau_{1} \cup \tau_{2}$ has the same direction label, then $e$ appears in only one of $\tau_{1}$ and $\tau_{2}$, and we delete it from that set. For any other pair of elements in $\tau_{1} \cup \tau_{2}$ with a common direction label, delete the element from $\tau_{1}$. Let $\tau_{1}^{\prime}$ and $\tau_{2}^{\prime}$ be the results of deleting elements from $\tau_{1}$ and $\tau_{2}$ respectively. Because $\tau_{1}$ is orthogonal, $\tau_{1}^{\prime}$ is a canonical sequence for $\sigma_{1}$. Because $e$ is a first/last element of $\sigma_{2}$, therefore $\tau_{2}^{\prime}$ is a canonical sequence for $\sigma_{2}$. 
At this point $\tau$ 's set of direction labels is equal to the union of the sets of direction labels of $\tau_{1}$ and $\tau_{2}$. We must verify that $\tau$ is a canonical sequence for $\sigma$. By construction, the elements of $\tau$ have distinct direction labels. Observe that by condition (2), the construction leaves $e$ in $\tau$ only if $e \in \tau_{1} \cap \tau_{2}$. Thus, by condition (1) and the fact that $e$ is a transition element, if a flat $F$ contains more than one element of $\tau$, then either $F$ is light, or $F \cap \tau$ is contained in $\sigma_{1}$ or $F \cap \tau$ is contained in $\sigma_{2}$, and in all three cases, $F \cap \tau$ has at most three elements, and they must be consecutive in $\sigma$.

\subsubsection{Canonical Sequences in Paths}

This section is about canonical sequences that can be found in simple shape paths. We need these results for the middle step of our plan: to break a simple shape cycle into paths, find canonical sequences in the paths and merge the canonical sequences. The main result of this section is the " $3 D$ U-Turn" Lemma that finds a canonical sequence of length four (including two opposite direction labels) in a simple shape path whose drawing leaves a plane and then returns to it. This case arises, for example, when we break a shape cycle at two transition vertices that lie in a plane.

We first recall the following result for $2 D$ shape paths from [4], with a slight change of notation.

Theorem 3 (2D Path [4]) Let $\sigma$ be a 2D shape path in the NSEW plane, and let $p$ be any point of the $N E$ quadrant. Then $\sigma$ admits a simple drawing $\Gamma(\sigma)$ that starts at the origin and that terminates at $p$ if and only if $\sigma$ contains a canonical sequence of type $\{N, E\}$.

Note that this theorem is stated for the $N E$ quadrant of the $N S E W$ plane, although we will apply it more generally, based on the discussion of symmetries at the end of Sect. 3. The same will be true of all results in this section: we will describe them for specific directions but apply them more generally.

We will find it convenient to have a weaker form of Theorem 2 (3D Path) where we specify only two of the direction relations:

Lemma 6 (Weak $3 D$ Path) Let $\Gamma(\sigma)$ be a simple drawing of a $3 D$ shape path $\sigma$ starting at vertex a located at the origin and reaching a vertex $u$ which is in the $U N E$ octant, or the $U N W$ octant, or the $U N$ quadrant. Then $\sigma$ contains a canonical sequence of type $\{U, N\}$.

Proof If $u$ lies in the $U N E$ quadrant, then by Theorem 2 (3D Path), $\sigma_{a u}$ contains a canonical sequence of type $\{U, N, E\}$, which is orthogonal, so we can remove the edge labeled $E$ to obtain a canonical sequence of type $\{U, N\}$. The argument for $u$ in the $U N W$ octant is similar.

Finally, if $u$ lies in the $U N$ quadrant, then $a$ and $u$ lie in the $N S U D$ plane, so by the general position assumption, $a$ and $u$ are joined by a path in that plane. By Theorem 3 (2D Path), $\sigma_{a u}$ contains a canonical sequence of type $\{U, N\}$.

Now we turn to the main focus of this section, which is a simple path whose drawing leaves a plane and then returns to it. We specify where the path returns as follows: 
Definition 7 Let $\Gamma(\sigma)$ be a drawing of a shape path $\sigma$. If $u v$ is an edge of $\sigma$ with $u$ in the $N E$ quadrant and $v$ in the $N W$ quadrant or in the $N$ axis, we say that $u v$ orthogonally intersects the $N$ axis. If $u$ lies in the $N E U$ octant and $v$ lies in the $N W U$ octant or in the $N U$ quadrant, we say that $u v$ orthogonally intersects the $N U$ quadrant.

Note that, by symmetry, this definition also applies to other quadrants and octants, including, for example, that edge $u v$ orthogonally intersects the $N$ axis when $u$ is in the $N W$ quadrant and $v$ is in the $N E$ quadrant or in the $N$ axis.

We first give a $2 D$ version of the result, where the path leaves the $N S$ axis and then returns to it.

Lemma 7 (2D U-Turn) Let $\Gamma(\sigma)$ be a simple drawing in the NSEW plane of a $2 D$ shape path $\sigma$ starting at vertex a located at the origin, with a later edge uv that orthogonally intersects the $N$ axis. Then $\sigma$ contains a canonical sequence of type $\{N, E, W\}$.

Proof By induction on $|\sigma|$, the length of $\sigma$. If $|\sigma|=3$, the lemma holds trivially. Now suppose that the lemma holds for $3 \leq|\sigma| \leq n$ and assume $|\sigma|=n+1$. If $u v$ is not the last edge of $\Gamma(\sigma)$, then we are done by induction. Moreover, if there is an edge different from $u v$ that orthogonally intersects the $N$ axis, then we are again done by induction. So we assume that $u v$ is the last edge of $\Gamma(\sigma)$ and that no other edge of $\Gamma(\sigma)$ orthogonally intersects the $N$ axis.

Let $a a^{\prime}$ denote the first edge of $\Gamma(\sigma)$. If the direction label of $a a^{\prime}$ is an $N$, then vertex $a^{\prime}$ is $S$ of $u v$ since $\Gamma(\sigma)$ is simple. Then we are done by applying induction on $\sigma_{a^{\prime} v}$. We now have the situation that the only point of $\sigma$ on the $N$ axis is the point where $u v$ intersects the $N$ axis.

Suppose that $u$ lies in the $N E$ quadrant (the case where $u$ lies in the $N W$ quadrant is the same after interchanging the direction labels $E$ and $W$ ). Let $b$ be the point where the $u v$ edge intersects the $N$ axis.

Consider the polygon formed by following $\sigma$ from $a$ to $u$ and adding the new edges $u b$ and $b a$. This is a simple polygon. The interior of the polygon is to the left as $\sigma$ is traversed, because the $N$ axis above $b$ is outside the polygon. Consider an Eastmost edge $e$ of the polygon. Then $e$ is an $N S$ edge, and the interior of the polygon lies to the west of $e$. Thus $e$ is an $N$ edge and, together with the edges of $\sigma$ before and after $e$, provides a canonical sequence of type $\{E, N, W\}$.

We now present the $3 D$ U-Turn Lemma. We find a canonical sequence of length four in a $3 D$ shape path with a simple drawing that leaves the $U D N S$ plane and then returns to it, say by orthogonally intersecting the $U N$ quadrant. One might hope that $\sigma$ would contain a canonical sequence of type $\{U, N, E, W\}$. (In fact, our conference paper claimed this.) However, this is false, as the example in Fig. 3 shows. For the shape cycle $\sigma=N U E S W$, a canonical sequence that contains $E$ and $W$ must also contain $S$. The best we can claim is that there is a canonical sequence whose set of direction labels contains $\{N, U, E, W\}$. Recall that we use the notation $\tau=A^{+}$to indicate that $\tau$ is a canonical sequence containing the elements $A$ plus possibly some other elements. 
Fig. 3 A shape path

$\sigma=e_{1} e_{2} e_{3} e_{4} e_{5}=N U E S W$

that orthogonally intersects the

$U N$ quadrant but does not

contain a canonical sequence of

type $\{E, W, U, N\}$. Note that in

the proof of Lemma 8 we will

translate the origin up to the

$N S E W$ plane containing $u v$

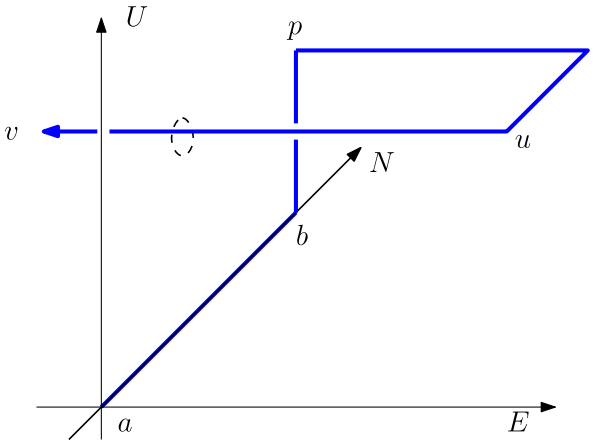

Lemma 8 (3D U-Turn) Let $\Gamma(\sigma)$ be a simple drawing of a $3 D$ shape path $\sigma$ starting at vertex a located at the origin, with a later edge uv that orthogonally intersects the NU quadrant. Then $\sigma$ contains a canonical sequence whose set of direction labels has $\{N, U, E, W\}$ as a subset, i.e. $\sigma$ contains a canonical sequence of type $\{N, U, E, W\}^{+}$.

Proof We will give an inductive proof of the "generic" version of the lemma that applies for other choices of directions. The induction is on $|\sigma|$, the length of $\sigma$. When $|\sigma|=4$, the lemma holds trivially. Suppose that the lemma holds for all values $4 \leq$ $|\sigma| \leq k$ and consider the case where $|\sigma|=k+1$. If $u v$ is not the last edge of $\sigma$, we are done by induction.

Let $p$ and $p^{\prime}$ be the first two vertices of the flat of $\sigma$ that contains $u v$. Denote the flat by $F=\sigma_{p v}$. Note that $F$ contains at least two edges $p p^{\prime}$ and $u v$.

We may assume without loss of generality that $F$ lies in an NSEW plane for the following reason. The edge $u v$ is either in an $N S E W$ or an $U D E W$ plane. If the latter, then we exchange labels $N, S$ with $U, D$; note that this does not affect whether sigma contains a canonical sequence of type $\{N, U, E, W\}^{+}$.

Most of our argument will be about $F$, so we will ease the notation by translating the origin up to the plane of $F$. Then $a$ lies in the $D$ axis, and $F$ orthogonally intersects the $N$ axis. Figure 3 does not reflect this assumption, but the forthcoming Fig. 4 does.

We assume without loss of generality that $u$ is in the $N E$ quadrant and that $v$ is in the $N W$ quadrant. In particular, we may stretch the edge $u v$ slightly to cross the axis without otherwise changing the drawing. If there exists an edge of $F$ other than $u v$ that orthogonally intersects the $N$ axis, we are done by induction.

The general idea is to break $\sigma$ into two subpaths, $\sigma_{1}$ from $a$ to $p$ or $p^{\prime}$ and $\sigma_{2} \subseteq F$ from $p$ or $p^{\prime}$ to $v$. Recall that we have translated the origin up to the plane of $F$. Depending on which quadrants of the NSEW plane $F$ visits, we deal with the following cases (listed here out of order): (3) if $F$ remains in the $N E$ quadrant, then we find a canonical sequence $U N E$ in $\sigma_{1}$ and $W$ in $F$; (1) if $F$ visits the $W$ half-plane, then at some point it must cross into the $E$ halfplane, so we can apply the planar Uturn result to find a canonical sequence $E W N$ in $F$, and combine it with a $U$ in $\sigma_{1}$; (2) otherwise, we are between these extremes and find $U E$ in $\sigma_{1}$ and $N W$ in $F$. The details are below. 

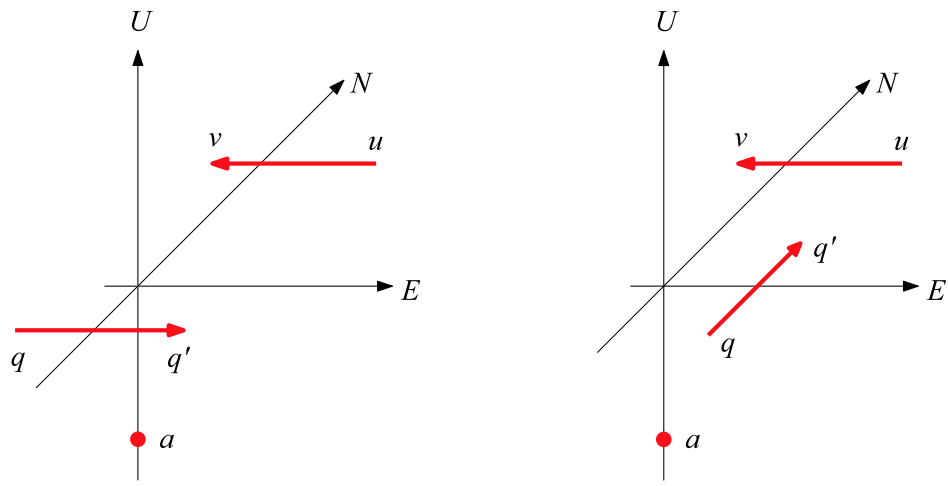

Fig. 4 Illustrating the $3 D$ U-Turn Lemma in Case 1 (left), where $\sigma_{p u}$ contains a vertex outside the $E$ halfplane, and Case 2 (right), where $\sigma_{p u}$ lies in the open $E$ halfplane and contains a vertex outside the $N E$ quadrant. Edges $u v$ and $q q^{\prime}$ lie in the NSEW plane

First we make some observations about vertices lying in axes. If a vertex $x$ of $F$ lies in the $N S$ axis or the $E W$ axis, then $a$ and $x$ lie in an isothetic plane, so by the general position assumption, there is a path in that plane from $a$ to $x$. Because $x$ is also in $F$, the remainder of $\sigma$ lies in the $N S E W$ plane of $F$. Thus $\sigma$ lies in two orthogonal planes whose line of intersection $l$ is the $N S$ axis or the $E W$ axis. Line $l$ either contains vertex $p$ and no other vertices, or the edge $p p^{\prime}$ and no other vertices. In particular, note that if $p^{\prime}$ lies in an axis, then so does $p$.

In Cases 1-3 below we will assume that $p$ does not lie in the $N$ axis North of $u v$, and therefore (by the observations above) that no vertex of $F$ lies in the $N$ axis North of $u v$. In Cases 1-3 we will always find a canonical sequence of type $\{E, W, U, N\}$, never a superset. Case 4 will deal with the situation where $p$ lies in the $N$ axis North of $u v$. This case, an example of which is shown in Fig. 3, is the only case where we need to resort to a superset of the desired directions.

Case 1: $\sigma_{p u}=F-v$ contains a vertex outside the open $E$ halfplane. See Fig. 4 (left). Then $F$ must enter the open $E$ halfplane, say at edge $q q^{\prime}$, with $q^{\prime}$ in the open $E$ halfplane. Either $q$ lies in the $W$ halfplane and $q q^{\prime}$ orthogonally intersects the $N S$ axis, or $q$ lies in the $N S$ axis. By assumption, no edge other than $u v$ orthogonally intersects the $N$ axis, and no vertex lies in the $N$ axis North of $u v$. Therefore $q q^{\prime}$ lies South of $u v$. By Lemma 7 ( $2 D$ U-Turn), the subpath $\sigma_{q v}$ contains a canonical sequence $\tau_{1}$ of type $\{E, W, N\}$. We consider two cases depending on whether $\sigma_{q v}$ contains the transition edge $p p^{\prime}$ or not.

(i) $\sigma_{q v}$ does not contain $p p^{\prime}$. Consider the subpath $\sigma_{a p}$, which is then separated from $\sigma_{q v}$ by transition edge $p p^{\prime}$, and which contains an element labeled $U$. By Lemma 3 (Basic Merge), $\sigma$ contains a canonical sequence of type $\{E, W, U, N\}$.

(ii) $\sigma_{q v}$ contains $p p^{\prime}$. Then $p p^{\prime}=q q^{\prime}$. Consider the subpath $\sigma_{a p^{\prime}}$, which shares the transition edge $p p^{\prime}$ with $\sigma_{q v}$. Because $p^{\prime}$ is in the $E$ halfplane, by Lemma 6 (Weak $3 D$ Path), $\sigma_{a p^{\prime}}$ contains a canonical sequence $\tau_{2}$ of type $U E$. Note that $\tau_{2}$ is orthogonal and that $\tau_{1}$ and $\tau_{2}$ both contain elements with direction label $E$, the direction of the shared edge $p p^{\prime}$. By Lemma 5 (Overlap Merge), $\sigma$ contains a canonical sequence of type $\{E, W, U, N\}$. 
Case 2: $\sigma_{p u}=F-v$ lies in the open $E$ halfplane and contains a vertex outside the (open) $N E$ quadrant. See Fig. 4 (right). Then $F$ must enter the $N E$ quadrant, say at edge $q q^{\prime}$, with $q^{\prime}$ in the $N E$ quadrant, and $q$ in the $S E$ quadrant or in the $E$ axis. By Theorem 3 (2D Path), the subpath $\sigma_{q v}$ contains a canonical sequence $\tau_{1}$ of type $\{N, W\}$. We consider two cases depending on whether $\sigma_{q v}$ contains the transition edge $p p^{\prime}$ or not.

(i) $\sigma_{q v}$ does not contain $p p^{\prime}$. Consider the subpath $\sigma_{a p}$ which is then separated from $\sigma_{q v}$ by transition edge $p p^{\prime}$. By Lemma 6 (Weak $3 D$ Path), $\sigma_{a p}$ contains a canonical sequence $\tau_{2}$ of type $\{U, E\}$. By Lemma 3 (Basic Merge), $\sigma$ contains a canonical sequence of type $\{E, W, U, N\}$.

(ii) $\sigma_{q v}$ contains $p p^{\prime}$. Then $p p^{\prime}=q q^{\prime}$. Consider the subpath $\sigma_{a p^{\prime}}$ which shares the transition edge $p p^{\prime}$ with $\sigma_{q v}$. By Theorem 2 (3D Path), $\sigma_{a p^{\prime}}$ contains a canonical sequence $\tau_{2}$ of type $U N E$. Note that $\tau_{2}$ is orthogonal and that $\tau_{1}$ and $\tau_{2}$ both contain elements with direction label $N$, the direction of the shared edge $p p^{\prime}$. By Lemma 5 (Overlap Merge), $\sigma$ contains a canonical sequence of type $\{E, W, U, N\}$.

Case 3: $\sigma_{p u}=F-v$ lies in the $N E$ quadrant. In fact, all we will use is that $p$ lies in the $N E$ quadrant. By Theorem 2 (3D Path), $\sigma_{a p}$ contains a canonical sequence of type $\{U, N, E\}$. Now $p \neq u$, since $F$ has at least two edges. Thus, the subpath $\sigma_{p^{\prime} v}$ contains $u v$, which has direction label $W$. The two subpaths are separated by transition edge $p p^{\prime}$, so by Lemma 3 (Basic Merge), $\sigma$ has a canonical sequence of type $\{E, W, U, N\}$.

Case 4: $p$ lies in the $N$ axis North of $u v$. In this case, as discussed at the beginning of the proof, $a$ and $p$ lie in a common $U D N S$ flat. We consider three cases depending on the position of $p^{\prime}$.

- If $p^{\prime}$ also lies in the $N$ axis, then, because $\sigma$ is simple, $p^{\prime}$ is also North of $u v$. By Theorem 3 (2D Path), $\sigma_{a p}$ contains a canonical sequence of type $\{N, U\}$. By Lemma 7 (2D U-Turn), $\sigma_{p^{\prime} v}$ contains a canonical sequence of type $\{E, W, S\}$. By Lemma 3 (Basic Merge), $\sigma$ has a canonical sequence of type $\{E, W, U, N, S\}$. We may not be able to remove the $S$.

- If $p^{\prime}$ lies in the $N W$ quadrant, then $F$ must orthogonally cross the $N$ axis to get from $p^{\prime}$ to $u$, contradiction to $u v$ being the first edge to orthogonally cross the $N$ axis.

- Otherwise $p^{\prime}$ lies in the $N E$ quadrant, and $p p^{\prime}$ has direction label $E$. Consider the subpaths $\sigma_{a p^{\prime}}$ and $\sigma_{p v}$ which overlap on transition edge $p p^{\prime}$. By Theorem 2 (3D Path), $\sigma_{a p^{\prime}}$ contains a canonical sequence $\tau_{1}$ of type $\{U, N, E\}$. By Lemma 7 (2D U-Turn), $\sigma_{p v}$ contains a canonical sequence $\tau_{2}$ of type $\{E, W, S\}$. Note that $\tau_{1}$ is orthogonal and that $\tau_{1}$ and $\tau_{2}$ both contain elements with direction label $E$, the direction of the shared edge $p p^{\prime}$. By Lemma 5 (Overlap Merge), $\sigma$ contains a canonical sequence of type $\{E, W, U, N, S\}$. We may not be able to remove the $S$.

These four cases complete the proof. 


\subsection{Proof of Necessity}

In this section we use the tools of the previous subsection to build up the proof of necessity. We show in a series of lemmas that if a simple drawing $\Gamma(\sigma)$ of a shape cycle $\sigma$ has a pair of transition vertices or edges in octant or quadrant relation, then $\sigma$ has a full canonical sequence. Finally, the main theorem is proved by arguing that $\Gamma(\sigma)$ always has at least one such pair of transition vertices or edges.

Our starting point, as mentioned in the introduction to Sect. 4, is the case of a pair of transition vertices in an octant relation. More generally, we may use a quadrant relation, and we may use transition edges instead of vertices.

Definition 8 If $x$ is a transition vertex or a transition edge, and $y$ is another transition vertex or transition edge, we say that $x$ and $y$ are in an octant relation in $\Gamma(\sigma)$ if no isothetic plane intersects both $x$ and $y$. Equivalently, they lie in opposite octants relative to some choice of origin.

Definition 9 If $x$ is a transition vertex or a transition edge, and $y$ is another transition vertex or transition edge, we say that $x$ and $y$ are in a quadrant relation in $\Gamma(\sigma)$ if they lie in a common isothetic plane, but no orthogonal isothetic plane intersects both. Equivalently, they lie in opposite quadrants of a common isothetic plane relative to some choice of origin.

Lemma 9 Let $\sigma$ be a shape cycle with a simple drawing $\Gamma(\sigma)$ that has two transition vertices/edges in an octant relation. Then $\sigma$ has a full canonical sequence.

Proof Let the transition vertices/edges be $x$ and $y$. Suppose without loss of generality that $x$ lies in the $U N E$ octant and $y$ lies in the $D S W$ octant. If $x$ [or $y$ ] is a transition vertex, cut $\sigma$ there. If $x$ [or $y$ ] is a transition edge, remove it to break $\sigma$. The result is two flat-separated subpaths $\sigma_{1}$ and $\sigma_{2}$, with $\sigma_{1}$ going from a point in the $U N E$ octant to a point in the $D S W$ octant, and $\sigma_{2}$ going from a point in the $D S W$ octant to a point in the $U N E$ octant. By Theorem 2 (3D Path), $\sigma_{1}$ has a canonical subsequence $\tau_{1}$ of type $\{D, S, W\}$, and $\sigma_{2}$ has a canonical subsequence $\tau_{2}$ of type $\{U, N, E\}$. By Lemma 3 (Basic Merge), $\tau_{1} \cup \tau_{2}$ is a canonical sequence for $\sigma$.

Next we extend Lemma 9 to the case where the two transition vertices/edges are only in a quadrant relation. We will need the $3 D$ U-Turn result (Lemma 8 ) for this case.

Lemma 10 Let $\sigma$ be a shape cycle with a simple drawing $\Gamma(\sigma)$ that has two transition vertices/edges in a quadrant relation. Then $\sigma$ has a full canonical sequence.

Proof Let the transition vertices/edges be $x$ and $y$. Suppose without loss of generality that $x$ and $y$ lie in the $U D N S$ plane and that $x$ lies in the $U N$ quadrant and $y$ lies in the $D S$ quadrant. Because $x$ and $y$ lie in a plane, the general position assumption implies that they are joined by a path in that plane. Suppose without loss of generality that it is the portion of $\sigma$ from $x$ to $y$ that lies in the $U D N S$ plane. If $x$ [or $y$ ] is a 
Fig. 5 If $a$ and $b$ lie on an isothetic line, then $\sigma$ splits into two flats

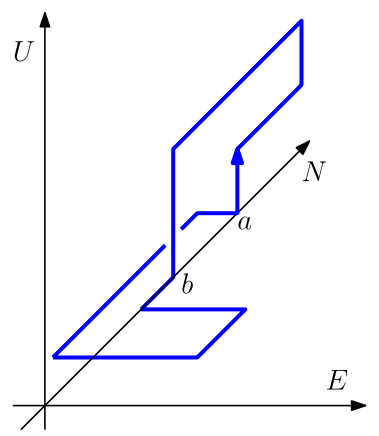

transition vertex, cut $\sigma$ there. If $x$ [or $y$ ] is a transition edge, remove it to break $\sigma$. The result is two flat-separated subpaths with $\sigma_{1}$ (say) lying in the $U D N S$ plane and going from a point in the $U N$ quadrant to a point in the $D S$ quadrant, and $\sigma_{2}$ going from a point in the $D S$ quadrant to a point in the $U N$ quadrant, with the first and last edges of $\sigma_{2}$ orthogonal to the $U D N S$ plane. By Theorem 3 (2D Path), $\sigma_{1}$ has a canonical sequence $\tau_{1}$ of type $\{D, S\}$. Note that $\tau_{1}$ is orthogonal. By Lemma 8 (3D U-Turn), $\sigma_{2}$ has a canonical sequence $\tau_{2}$ of type $\{E, W, U, N\}^{+}$. By Lemma 4 (Merge), $\sigma$ has a full canonical sequence.

Finally, we deal with the somewhat degenerate case of non-adjacent vertices that lie in a common isothetic line. By the general position assumption, $\sigma$ then lies in the union of two orthogonal planes.

Lemma 11 Let $\sigma$ be a shape cycle with a simple drawing $\Gamma(\sigma)$ that has two vertices, $a$ and $b$, not joined by an edge, lying in a common isothetic line. Then $\sigma$ has a full canonical sequence.

Proof Suppose without loss of generality that $a$ and $b$ lie in the $N$ axis. By Lemma 2 (General Position), $\sigma$ splits into two flats that lie in the $U D N S$ plane and the $N S E W$ plane, respectively. See Fig. 5.

Each of $a$ and $b$ is either a transition vertex - in which case we cut $\sigma$ at the transition vertex - or is incident to a transition edge that lies in the $N$ axis-in which case we break $\sigma$ by removing the transition edge. The result is two flat-separated subpaths, $\sigma_{1}$ in the $U D N S$ plane and $\sigma_{2}$ in the $N S E W$ plane, each going from a point in the $N$ axis to a last edge that orthogonally intersects the $N$ axis. Suppose without loss of generality that $\sigma_{1}$ 's start point is North of its end point. By Lemma 7 (2D U-Turn), $\sigma_{1}$ has a canonical sequence of type $\{U, D, S\}$, and $\sigma_{2}$ has a canonical sequence of type $\{E, W, N\}$. By Lemma 3 (Basic Merge), $\sigma$ has a full canonical sequence.

We need to examine two more configurations of pairs of transition edges. The first is the case of parallel transition edges that do not lie in a common isothetic plane, and the second is defined as follows. We say that two transition edges $a a^{\prime}$ and $b b^{\prime}$ are in a skew relation in $\Gamma(\sigma)$ if, relative to some choice of origin and some permutation of directions, $a a^{\prime}$ lies in the $D S$ quadrant, and $b b^{\prime}$ orthogonally intersects the $U N$ 
Fig. 6 Example of edges in a skew relation: $a a^{\prime}$ lies in the $S$ axis, and $b b^{\prime}$ orthogonally intersects the $U N$ quadrant

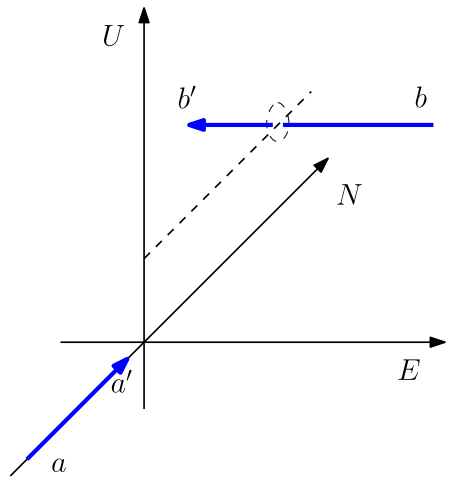

quadrant. Equivalently, by further translation of the origin and permutation of the directions, $a a^{\prime}$ lies in the $S$ axis, and $b b^{\prime}$ orthogonally intersects the $U N$ quadrant. See Fig. 6. With this definition in mind, we immediately consider this case and return afterwards to the former case.

Observe that until now we only needed to merge canonical sequences of disjoint subpaths. However, for the final two configurations of pairs of transition edges, we will need the full power of the merging lemma that deals with subpaths that overlap on a transition edge.

Lemma 12 Let $\sigma$ be a shape cycle with a simple drawing $\Gamma(\sigma)$ that has two transition edges, $a a^{\prime}$ and $b b^{\prime}$, in a skew relation. Then $\sigma$ has a full canonical sequence.

Proof Suppose without loss of generality that $a a^{\prime}$ lies in the $S$ axis and $b b^{\prime}$ orthogonally intersects the $U N$ quadrant. Furthermore, suppose without loss of generality that $b b^{\prime}$ has direction label $W$, i.e. that $b$ lies in the $U N E$ octant and $b^{\prime}$ lies in the $U N W$ octant or in the $U N$ quadrant.

Consider the subpaths $\sigma_{b a}$ and $\sigma_{a^{\prime} b^{\prime}}$. The subpaths share the edge $b b^{\prime}$ but are otherwise flat-separated (i.e. $\sigma_{b a}-b b^{\prime}$ and $\sigma_{a^{\prime} b^{\prime}}-b b^{\prime}$ are flat-separated). By Theorem 2 (3D Path), $\sigma_{b a}$ contains a canonical sequence $\tau_{1}$ of type $\{S, D, W\}$. Note that $\tau_{1}$ is orthogonal. By Lemma 8 (3D U-Turn), $\sigma_{a^{\prime} b^{\prime}}$ contains a canonical sequence $\tau_{2}$ of type $\{E, W, U, N\}^{+}$. Note that $\tau_{1}$ and $\tau_{2}$ both contain elements with direction label $W$ which is the label of the shared edge $b b^{\prime}$. Also note that $\tau_{1} \cup \tau_{2}$ contains all 6 direction labels. Thus by Lemma 5 (Overlap Merge), $\sigma$ has a full canonical sequence.

Finally, we consider transition edges that are parallel but do not lie in a common isothetic plane. This proof is the trickiest one, but we need it because a shape cycle may have all its transition edges parallel to each other.

Lemma 13 Let $\sigma$ be a shape cycle with a simple drawing $\Gamma(\sigma)$ that has two transition edges $a a^{\prime}$ and $b b^{\prime}$ that are parallel but do not lie in an isothetic plane. Then $\sigma$ has a full canonical sequence.

Proof Suppose without loss of generality that $a a^{\prime}$ and $b b^{\prime}$ are $E W$ edges. If they lie in an octant relation, we may apply Lemma 9 to obtain a full canonical sequence. 
Thus, we may assume that they both intersect an $U D N S$ plane. Choose such a plane and translate the origin to the point where $a a^{\prime}$ intersects the plane. Furthermore, we may assume without loss of generality that $b b^{\prime}$ intersects the $U N$ quadrant of this plane.

We begin by dealing with the case that two of $a, a^{\prime}, b, b^{\prime}$ lie on a common $U D N S$ plane. Suppose that one of $a, a^{\prime}$, say $\alpha$, and one of $b, b^{\prime}$, say $\beta$, lie on a common $U D N S$ plane. By the general position assumption, there is a path joining $\alpha$ and $\beta$. This path cannot be a single edge, otherwise $a a^{\prime}$ and $b b^{\prime}$ would lie in a common plane. Therefore, the path forms a flat. We claim that $\alpha$ and $\beta$ are then transition vertices, since each one is incident to two transition edges, the one in the UDNS plane and the $E W$ edge $a a^{\prime}$ or $b b^{\prime}$. Therefore by Lemma $10, \sigma$ has a full canonical sequence.

We may therefore assume that no two of $a, a^{\prime}, b, b^{\prime}$ lie on a common $U D N S$ plane. We will say that vertex $x$ is east of vertex $y$ if $x$ is east of the $U D N S$ plane through $y$. Suppose without loss of generality that the $b b^{\prime}$ edge has the easternmost vertex (otherwise rename $a a^{\prime}$ and $b b^{\prime}$ ). Suppose that the $b b^{\prime}$ edge is labeled $W$ (otherwise reverse the cycle) - thus the easternmost vertex is $b$, and both $a$ and $a^{\prime}$ are $D S W$ of $b$.

Case 1: $a^{\prime}$ is East of $b^{\prime}$. Note that $a a^{\prime}$ may be labeled $W$ or $E$. Consider the subpaths $\sigma_{a^{\prime} b^{\prime}}$ and $\sigma_{b a}$. These subpaths share the transition edge $b b^{\prime}$ but are otherwise flatseparated (i.e. $\sigma_{a^{\prime} b^{\prime}}-b b^{\prime}$ and $\sigma_{b a}-b b^{\prime}$ are flat-separated). By Lemma 8 (3D UTurn), $\sigma_{a^{\prime} b^{\prime}}$ contains a canonical sequence $\tau_{1}$ of type $\{E, W, U, N\}^{+}$. By Theorem 2 (3D Path), $\sigma_{b a}$ contains a canonical sequence $\tau_{2}$ of type $\{D, S, W\}$. Note that $\tau_{2}$ is orthogonal,and that $\tau_{1}$ and $\tau_{2}$ both contain elements with direction label $W$, which is the label of the shared edge $b b^{\prime}$. Also note that $\tau_{1} \cup \tau_{2}$ contains all 6 direction labels. Thus by Lemma 5 (Overlap Merge), $\sigma$ has a full canonical sequence.

Case 2: $a^{\prime}$ is West of $b^{\prime}$. In this case $a$ must be East of $b^{\prime}$ since the edges intersect a common $U D N S$ plane. Therefore $a a^{\prime}$ is labeled $W$. See Fig. 7. Consider the subpath $\sigma_{a b^{\prime}}$. By Lemma 8 (3D U-Turn), $\sigma_{a b^{\prime}}$ contains a canonical sequence $\tau_{1}$ of type $\{E, W, U, N\}^{+}$. Now $\tau_{1}$ cannot use both $a a^{\prime}$ and $b b^{\prime}$ since they are both $W$ edges. We consider two cases depending on which edge is not in $\tau_{1}$.

(i) $\tau_{1}$ does not contain $a a^{\prime}$. Then $\tau_{1}$ lies in the subpath $\sigma_{1}=\sigma_{a b^{\prime}}-\left\{a a^{\prime}\right\}=\sigma_{a^{\prime} b^{\prime}}$. Consider the subpath $\sigma_{2}=\sigma_{b a}$. Then $\sigma_{1}$ and $\sigma_{2}$ share the edge $b b^{\prime}$ but are otherwise flat-separated (i.e. $\sigma_{1}-b b^{\prime}$ and $\sigma_{2}-b b^{\prime}$ are flat-separated). By Theorem 2

Fig. 7 Parallel transition edges $a a^{\prime}$ and $b b^{\prime}$ in Case 2 of the proof of Lemma 13

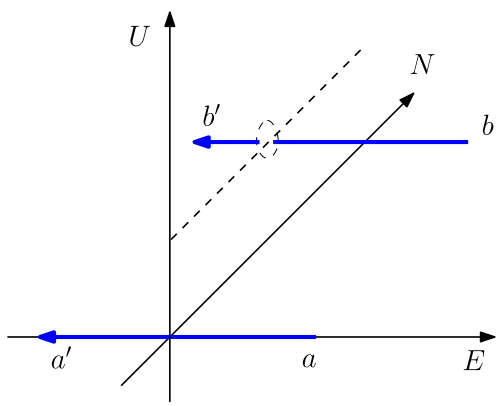


(3D Path), $\sigma_{2}$ contains a canonical subsequence $\tau_{2}$ of type $\{D, S, W\}$. Note that $\tau_{2}$ is orthogonal and that $\tau_{1}$ and $\tau_{2}$ both contain elements with direction label $W$, which is the label of the shared edge $b b^{\prime}$. Also note that $\tau_{1} \cup \tau_{2}$ contains all six direction labels. Thus by Lemma 5 (Overlap Merge), $\sigma$ has a full canonical sequence.

(ii) $\tau_{1}$ does not contain $b b^{\prime}$. This case is similar. Then $\tau_{1}$ lies in the subpath $\sigma_{1}=$ $\sigma_{a b^{\prime}}-\left\{b b^{\prime}\right\}=\sigma_{a b}$. Consider the subpath $\sigma_{2}=\sigma_{b^{\prime} a^{\prime}}$. Then $\sigma_{1}$ and $\sigma_{2}$ share the edge $a a^{\prime}$ but are otherwise flat-separated (i.e. $\sigma_{1}-a a^{\prime}$ and $\sigma_{2}-a a^{\prime}$ are flatseparated). By Theorem 2 ( $3 D$ Path), $\sigma_{2}$ contains a canonical subsequence $\tau_{2}$ of type $\{D, S, W\}$. Note that $\tau_{2}$ is orthogonal and that $\tau_{1}$ and $\tau_{2}$ both contain elements with direction label $W$, which is the label of the shared edge $a a^{\prime}$. Also note that $\tau_{1} \cup \tau_{2}$ contains all six direction labels. Thus by Lemma 5 (Overlap Merge), $\sigma$ has a full canonical sequence.

We have now completed our series of geometric lemmas. It remains to prove that any simple drawing of a shape cycle has a pair of transition vertices/edges in one of the above configurations. We begin with one structural result.

Lemma 14 Let $\sigma$ be a simple shape cycle that has a heavy flat $F$ whose transition edges have orthogonal directions. Then $\sigma$ has a full canonical sequence.

Proof Suppose that $F$ has transition edges $a a^{\prime}$ and $b b^{\prime}$. (We do not specify which edge is at which end of $F$.) Consider a simple drawing $\Gamma(\sigma)$, and suppose without loss of generality that $F$ lies in the $U D N S$ plane through the origin and that $a a^{\prime}$ is an $U D$ edge with $a$ in the $U$ axis and $b b^{\prime}$ is a $N S$ edge with $b$ in the $S$ axis. It cannot be the case that both $a a^{\prime}$ and $b b^{\prime}$ contain the origin ( $F$ is a heavy flat and the drawing is simple), so suppose without loss of generality that $b^{\prime}$ lies in the $S$ axis.

If $a^{\prime}$ lies in the $U$ axis, then $a a^{\prime}$ and $b b^{\prime}$ are in a quadrant relation, and we are done by Lemma 10. If $a^{\prime}$ lies at the origin, then $a^{\prime}$ and $b$ are non-adjacent vertices lying in the $N S$ axis, so we are done by Lemma 11 .

Thus we may suppose that $a^{\prime}$ lies in the $D$ axis. Consider the other flat $F^{\prime}$ that contains the transition edge $a a^{\prime}$. Then $F^{\prime}$ lies in the $U D E W$ plane through the origin. Let $c c^{\prime}$ be its other transition edge. Note that $F^{\prime}$ may be a light flat, so $c c^{\prime}$ may be incident to $a a^{\prime}$. If $c c^{\prime}$ lies completely in one quadrant of this plane, then $b b^{\prime}$ and $c c^{\prime}$ are in a full octant relation, so we are done by Lemma 9. If $c c^{\prime}$ lies in either the $U D$ or $E W$ axis, then, because it cannot contain the origin, $b b^{\prime}$ and $c c^{\prime}$ are in a quadrant relation, and we can apply Lemma 10. Otherwise $c c^{\prime}$ orthogonally intersects the $U D$ axis or the $E W$ axis, so $b b^{\prime}$ and $c c^{\prime}$ are in a skew relation, and we can apply Lemma 12. See Fig. 8.

Theorem 4 (Necessity) Every 3D simple shape cycle contains a full canonical sequence.

Proof Let $\sigma$ be a shape cycle with a simple drawing $\Gamma(\sigma)$. Suppose first that $\sigma$ has a heavy flat $F$. If $F$ has transition vertices at both ends, then those transition vertices 
Fig. 8 Orthogonal transition edges $a a^{\prime}$ and $b b^{\prime}$ in a heavy flat for the proof of Lemma 14

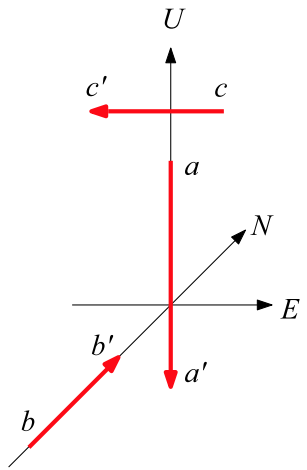

either lie in an isothetic line, and we apply Lemma 11, or they are in a quadrant relation, and we apply Lemma 10.

Otherwise, $F$ has a transition edge $f$ that is in another heavy flat $F^{\prime}$. Suppose that $F$ has transition edges $e$ and $f$ and that $F^{\prime}$ has transition edges $f$ and $g$. If either $e$ or $g$ is orthogonal to $f$, we apply Lemma 14. Otherwise $e$ and $g$ are both parallel to $f$. Note that $F$ and $F^{\prime}$ lie in orthogonal planes, $f$ lies in the line of intersection of those planes, and $e$ and $g$ do not lie in that line (otherwise we have non-adjacent vertices in an isothetic line and can apply Lemma 11). Thus $e$ and $g$ do not share an isothetic plane. The result then follows from Lemma 13.

We are left with the case where $\sigma$ has only light flats. Then every vertex is a transition vertex. Because $\sigma$ has at least six vertices, there are two vertices, $a$ and $b$, separated by at least three edges. Then $a$ and $b$ are in an octant relation, and we are done by Lemma 9.

\section{Sufficiency}

In this section, we prove that any shape cycle $\sigma$ with a full canonical sequence admits a simple drawing $\Gamma(\sigma)$. The proof is constructive, giving an algorithm for computing $\Gamma(\sigma)$.

We first give an overview of the construction technique in Sect. 5.1. As a main tool, we prove that if $\sigma$ has a full canonical sequence, then it has a proper full canonical sequence. Then in Sect. 5.2 we use such a proper full canonical sequence to construct a simple drawing of a shape cycle.

\subsection{Overview of Construction}

The intuition behind our construction of a simple drawing $\Gamma(\sigma)$ for a shape cycle $\sigma$ is to imagine the drawing as an elaboration of a cycle with six relatively long canonical edges drawn along the edges of a rectangular box in $3 D$. Observe that, if a shape cycle $\sigma$ has length exactly six, any simple drawing of $\sigma$ has one of two essentially different shapes, namely, a chair shape such as $N U S W D E$ or a skew shape such as $N U W S D E$. Both the chair and the skew shape can be drawn along the edges of a rectangular box, as shown in Fig. 9. 
Fig. 9 (a) Chair shape;

(b) skew shape

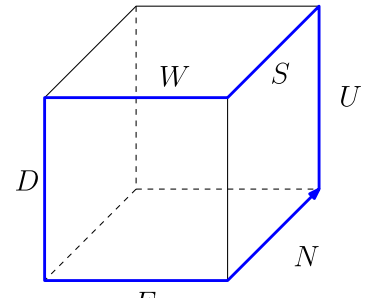

(a)

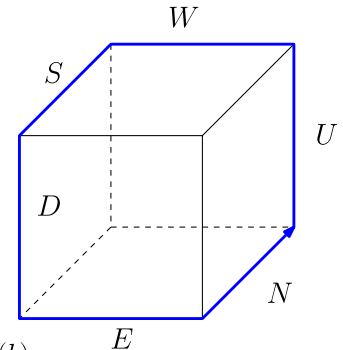

(b)

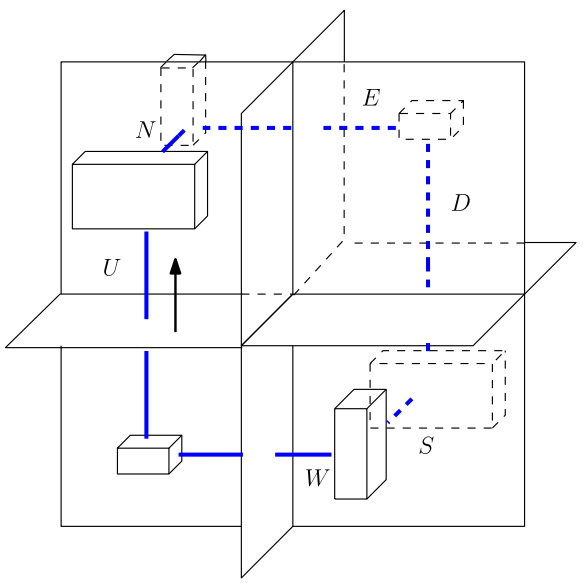

(b)

Fig. 10 A schematic drawing for $\Gamma(\sigma)$ for (a) a chair shape with a canonical sequence $U E N D S W$ and (b) a skew shape with a canonical sequence $U N E D S W$. Connector paths lie inside the boxes

More generally, if $\sigma$ has length greater than six, then the full canonical sequence in $\sigma$ provides the six edges of a chair shape or a skew shape which we draw along the edges of a rectangular box. The remaining elements of $\sigma$ form connector paths which we draw as paths of short edges located near the vertices of the box. By making the canonical edges sufficiently long, we can make the connector paths lie in distinct octants, thereby avoiding any collisions among them. Our plan therefore is to first draw the connector paths and then assign lengths to the canonical edges so that the connector paths lie in distinct octants. For an example, see Fig. 10.

One difficulty with this approach is that there may be adjacent elements in a canonical sequence that are oppositely directed-such a canonical sequence does not provide a chair or skew shape. This motivates the following definition.

Definition 10 A subsequence $\tau$ of a shape cycle $\sigma$ is a proper full canonical sequence if $\tau$ is a full canonical sequence and no two elements that are adjacent in $\tau$ are oppositely directed. 
In the remainder of this section we show that the existence of a full canonical sequence implies the existence of a proper full canonical sequence. Our proof is constructive and works by applying a sequence of editing operations. In the following Sect. 5.2 we give the algorithm to construct a simple drawing starting from a proper full canonical sequence.

Lemma 15 If a shape cycle $\sigma$ contains a full canonical sequence, then $\sigma$ contains $a$ proper full canonical sequence.

Proof Let $\tau$ be a full canonical sequence for $\sigma$. If $\tau$ is also proper, we are done. Otherwise, $\tau$ contains between one and three pairs of oppositely directed elements that are adjacent in $\tau$. We will show that this number can always be reduced. The idea is to look between such a pair in $\sigma$ (where they are not adjacent) and find an element that we can swap in to $\tau$, while swapping out the old element of $\tau$ with the same direction label. We must be careful to maintain the properties of a canonical sequence. In particular, the only problem with removing an element of $\tau$ is when it is the middle element of three elements that lie in a common flat of $\sigma$, so this is one situation we would like to avoid. We now elaborate on this plan. We consider two cases depending on whether $\tau$ contains more than one, or exactly one pair of oppositely directed adjacent elements.

Case 1: Suppose that $\tau$ contains two or three pairs of oppositely directed adjacent elements. Any such pair cannot be adjacent in $\sigma$ and therefore cannot share a flat in $\sigma$. We may assume without loss of generality that $U D$ and $E W$ are such pairs and that $\tau=\bar{U} \bar{D} \bar{N} \bar{S} \bar{E} \bar{W}$ or $\tau=\bar{U} \bar{D} \bar{N} \bar{E} \bar{W} \bar{S}$.

We first prove that no flat of $\sigma$ contains three elements of $\tau$. Three elements of $\tau$ from one flat must be consecutive in $\sigma$ and must therefore consist of an oppositely directed pair separated by one other element. For example, $N E S$ is possible, but $N S E$ is not. Observe that this pattern does not occur in either case of $\tau$ : the pairs $\bar{U}, \bar{D}$ and $\bar{E}, \bar{W}$ appear consecutively, and the elements $\bar{N}, \bar{S}$ are either consecutive or separated by two other elements. Therefore no flat of $\sigma$ contains three elements of $\tau$.

Let $\bar{X}$ denote the element that immediately follows $\bar{U}$ in $\sigma$. Let $\hat{X}$ denote the element of $\tau$ that has the same direction label as $\bar{X}$. We will show that replacing $\hat{X}$ by $\bar{X}$ in $\tau$ yields a better canonical sequence. First note that $\tau^{\prime}=\tau-\{\hat{X}\}$ is canonical since no flat of $\sigma$ contains three elements of $\tau$. Next we show that $\tau^{\prime \prime}=\tau^{\prime} \cup\{\bar{X}\}$ is canonical. We only need to show that $\bar{X}$ does not share a flat with a non-adjacent element of $\tau^{\prime} . \bar{X}$ lies between $\bar{U}$ and $\bar{D}$. It is adjacent to $\bar{U}$. If $\bar{X}$ and $\bar{D}$ share a flat, then $\bar{U}$ is also in this flat since $\bar{U}$ lies in the plane of $\bar{X}$ and $\bar{D}-$ but $\bar{U}$ and $\bar{D}$ do not share a flat. Thus $\tau^{\prime \prime}$ is a full canonical sequence. It has fewer pairs of adjacent oppositely directed elements.

Case 2: Suppose that $\tau$ contains exactly one pair of oppositely directed adjacent elements. We may then assume without loss of generality that $\tau=\bar{U} \bar{D} \bar{N} \bar{E} \bar{S} \bar{W}$. Note that, as in Case 1 , the two elements $\bar{U}$ and $\bar{D}$ belong to distinct flats.

Recall that, by definition, a canonical sequence does not contain four elements from the same flat. If three elements of $\tau$ belong to the same flat, they must be either 
$\bar{N} \bar{E} \bar{S}$ or $\bar{E} \bar{S} \bar{W}$. Therefore, regardless of whether there is a flat containing three elements of $\tau$ or not, each of $\tau-\{\bar{N}\}, \tau-\{\bar{W}\}$, and $\tau-\{\bar{E}, \bar{S}\}$ is canonical and still has only one pair of oppositely directed adjacent elements.

As in Case 1 , let $\hat{X}$ be the element after $\bar{U}$ in $\sigma$, and let $\bar{X}$ be the element of $\tau$ with the same direction label as $\hat{X}$. Similarly, let $\hat{Y}$ be the element before $\bar{D}$ in $\sigma$, and let $\bar{Y}$ be the element of $\tau$ with the same direction label as $\hat{Y}$.

If $\hat{X}$ has direction $N$ or $W$, then $\tau^{\prime \prime}=\tau-\{\bar{X}\} \cup\{\hat{X}\}$ is a proper full canonical sequence by the same argument as in Case 1. Similarly, if $\hat{Y}$ has direction $N$ or $W$, then $\tau^{\prime \prime}=\tau-\{\bar{Y}\} \cup\{\hat{Y}\}$ is a proper full canonical sequence. So we assume that each of $\hat{X}$ and $\hat{Y}$ has direction $E$ or $S$. There are two cases to consider.

(i) $\hat{X}$ and $\hat{Y}$ have different directions. If $\hat{X}$ and $\hat{Y}$ do not share a flat, then $\tau^{\prime \prime}=$ $\tau-\{\bar{X}, \bar{Y}\} \cup\{\hat{X}, \hat{Y}\}$ is a proper full canonical sequence. So suppose that $\hat{X}$ and $\hat{Y}$ share a flat $F$. Then $F$ is an $E W N S$ flat with transition elements $\hat{X}$ and $\hat{Y}$-in particular, neither $\bar{U}$ nor $\bar{D}$ share a flat with any element of $F$ except $\hat{X}$ and $\hat{Y}$, respectively. Let $\hat{Z}$ be the element after $\hat{X}$ in $\sigma$, and let $\bar{Z}$ be the element of $\tau$ that has the same direction label as $\hat{Z}$. Note that we allow the possibility that $\hat{Z}=\hat{Y}$. If $\hat{Z}$ has direction label $N$ or $W$, then $\tau^{\prime \prime}=\tau-\{\bar{Z}\} \cup\{\hat{Z}\}$ is a proper full canonical sequence. Otherwise $\hat{Z}$ has direction label $E$ or $S$ and different from $\hat{X}$, so $\tau^{\prime \prime}=\tau-\{\bar{X}, \bar{Z}\} \cup\{\hat{X}, \hat{Z}\}$ is a proper full canonical sequence.

(ii) $\hat{X}$ and $\hat{Y}$ have the same direction label. Suppose without loss of generality that it is $E$. Let $F$ be the flat containing $\bar{U}$ and $\hat{X}$. This is a $U D E W$ flat. Let $\hat{Z}$ be the first element of $\sigma$ after flat $F$, and let $\bar{Z}$ be the element of $\tau$ that has the same direction label at $\hat{Z}$. Then $\hat{Z}$ has direction $N$ or $S$. Note that $\hat{Z}$ and $\bar{D}$ do not share a flat: it would be an NSUD flat but contains $\hat{Y}$ which has direction $E$. If $\hat{Z}$ has direction $N$, then $\tau^{\prime \prime}=\tau-\{\bar{Z}\} \cup\{\hat{Z}\}$ is a proper full canonical sequence. Otherwise $\hat{Z}$ has direction $S$, and $\tau^{\prime \prime}=\tau-\{\bar{X}, \bar{Z}\} \cup\{\hat{X}, \hat{Z}\}$ is a proper full canonical sequence (note that if $\hat{X}$ and $\hat{Z}$ share a flat, then they are adjacent).

This completes the proof.

\subsection{Constructing a Simple Drawing}

In this section we give a constructive proof for the existence of a simple drawing $\Gamma(\sigma)$ for any shape cycle $\sigma$ with a proper full canonical sequence. Specifically, we give a linear time algorithm to construct $\Gamma(\sigma)$. The algorithm follows the plan outlined at the beginning of the previous section. First, in Sect. 5.2.1, we give an algorithm to draw the connector paths, making use of our previous work on drawing shape paths. Then, in Sect. 5.2.2, we compute lengths for the canonical edges by solving a system of equations that capture the conditions that (1) the connector paths are placed in distinct octants and (2) the overall cycle closes. Correctness of the construction is addressed in Sect. 5.2.3.

\subsubsection{Drawing of Connectors}

We first recall a method from [4] for constructing drawings of simple shape paths, which will be used to draw the connectors. 
Definition 11 [4] A drawing $\Gamma(\sigma)$ of a shape path $\sigma$ is an expanding drawing if each segment travels one unit farther in its assigned direction than the extreme points, with respect to that direction, of the previous segments of $\Gamma(\sigma)$. A drawing $\Gamma(\sigma)$ of a shape path $\sigma$ is a doubly expanding drawing if its first and last edges can be replaced by arbitrarily long edges without creating any intersections within the drawing of that shape path.

Lemma 16 [4] Any simple shape path $\sigma$ admits an expanding drawing that can be computed in linear time. Moreover, if $\sigma$ contains at least two flats, or $|\sigma|=2$, then $\sigma$ admits a doubly expanding drawing that can be computed in linear time.

In this section, we show how to construct a simple drawing for each connector in a shape cycle $\sigma$. By Lemma 15, we assume that the given full canonical sequence $\tau$ is proper. Let $\bar{X}$ and $\bar{Y}$ denote two canonical elements that are adjacent in $\tau$. Since $\tau$ is proper, $\bar{X}$ and $\bar{Y}$ are orthogonal to each other.

Consider the sequence of elements in $\sigma$, denoted by $\sigma_{\bar{X}} \bar{Y}$, from $\bar{X}$ to $\bar{Y}$, inclusive. Thus $\sigma_{\bar{X}} \bar{Y}$ consists of the connector path plus the canonical edge at either end. Observe that, since $\bar{X}$ and $\bar{Y}$ are canonical elements, either they belong to the same flat (in which case $\left|\sigma_{\bar{X}} \bar{Y}\right|=2$ ), or $\sigma_{\bar{X}} \bar{Y}$ contains at least two flats. Therefore, by Lemma 16, $\sigma_{\bar{X}} \bar{Y}$ admits a doubly expanding drawing $\Gamma\left(\sigma_{\bar{X}} \bar{Y}\right)$. Below, we briefly review how to construct this drawing. Note that we assign temporary lengths to $\bar{X}$ to $\bar{Y}$ during this process, but their final lengths are determined later on.

The case where $\sigma_{\bar{X}} \bar{Y}$ has exactly two elements is clear.

If $\sigma_{\bar{X}} \bar{Y}$ has more than two elements, we complete the drawing in two parts. Let $\sigma_{\bar{X}} \bar{Y}$ have the form $\bar{X} \ldots \hat{Z} \hat{Z}^{\prime} \ldots \bar{Y}$, where $\hat{Z}$ is the transition element, distinct from $\bar{X}$, of the first flat of $\sigma_{\bar{X}} \bar{Y}$. Then $\hat{Z}^{\prime}$ is the first edge that leaves this flat. We first construct the drawing of $\sigma_{\bar{X}} \hat{Z}$ and then add the drawing of $\sigma_{\hat{Z}^{\prime} \bar{Y}}$.

Starting at the origin, create a singly expanding drawing for the sequence $\hat{Z} \ldots \bar{X}$ obtained by traversing the elements of $\sigma_{\bar{X}} \hat{Z}$ in the negative sense, from $\hat{Z}$ back to $\bar{X}$. A singly expanding drawing of $\hat{Z} \ldots \bar{X}$ can be constructed by iteratively assigning each edge a length such that it protrudes distance 1 outside the bounding box of the edges already created. For example, consider the sequence $\sigma_{\bar{X}} \bar{Y}=$ $\bar{N} W N E S W S \hat{E} \hat{U} N D S W U \bar{E}$, with the first flat $\bar{N} W N E S W S \hat{E}$. Figure 11 shows the singly expanding drawing for the first flat $\sigma_{\bar{N} \hat{E}}$.

Fig. 11 An expanding drawing for $\bar{N} W N E S W S \hat{E}$

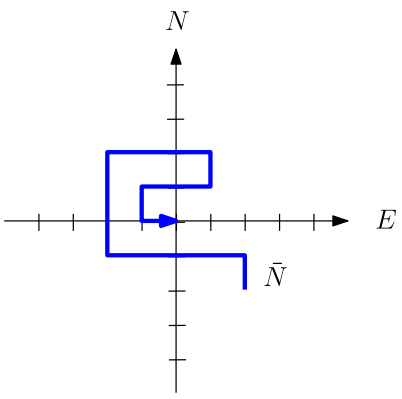


Fig. 12 A doubly expanding drawing for a connector $\bar{N} W N E S W S \hat{E} \hat{U} N D S W U \bar{E}$

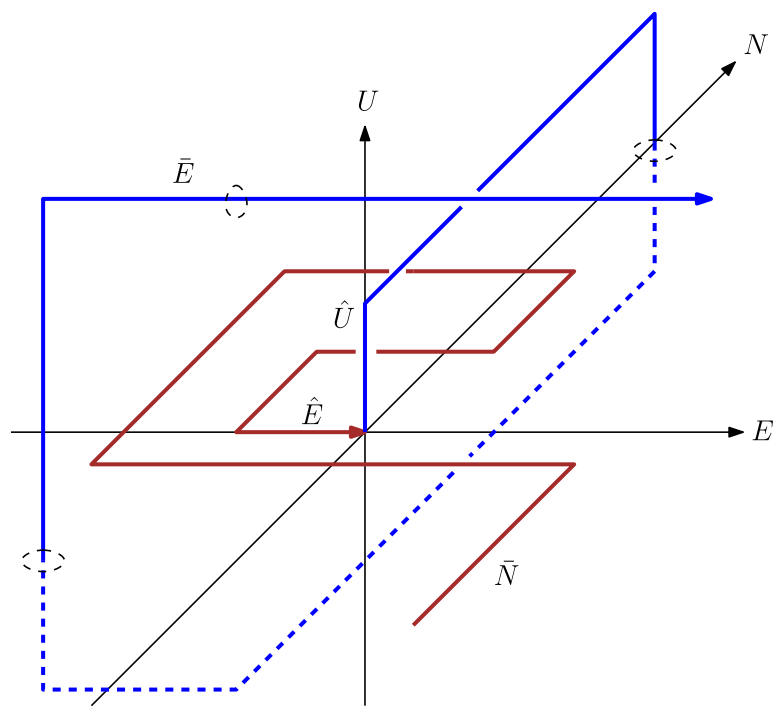

Finally, complete the drawing of $\sigma_{\bar{X}} \bar{Y}$ by constructing a singly expanding drawing of $\hat{Z}^{\prime} \ldots \bar{Y}$. Starting at the origin, iteratively assign each edge of $\hat{Z}^{\prime} \ldots \bar{Y}$ such that its tip protrudes distance 1 outside the bounding box of all the edges created so far, including the edges of $\Gamma\left(\sigma_{\bar{X}} \hat{Z}\right)$. Figure 12 shows a doubly expanding drawing of a connector $\sigma_{\bar{X} \bar{Y}}=\bar{N} W N E S W S \hat{E} \hat{U} N D S W U \bar{E}$.

As shown in [4], the drawing $\Gamma\left(\sigma_{\bar{X}} \bar{Y}\right)$ thus obtained has the doubly expanding property, i.e. the drawing remains simple regardless of the lengths assigned to $\bar{X}$ and $\bar{Y}$. Therefore, we may compute a doubly expanding drawing for each connector and freely assign lengths to the canonical edges $\bar{X}$ and $\bar{Y}$ without intersecting edges in the connectors.

\subsubsection{Drawing of Canonical Edges}

We first determine the lengths of the canonical edges in $\Gamma(\sigma)$. From these we determine the coordinates of all vertices in the drawing. To ease notation, we shall only describe how to determine the lengths of $\bar{U}$ and $\bar{D}$. The lengths of the remaining canonical edges can be determined in a similar fashion. See Fig. 10 for a schematic drawing for $\Gamma(\sigma)$.

Write $\sigma$ as $\bar{U} \tau \bar{D} \beta$. In other words, $\tau$ (for "top") denotes the subpath of $\sigma$ from $\bar{U}$ to $\bar{D}$, and $\beta$ (for "bottom") denotes the subpath of $\sigma$ from $\bar{D}$ to $\bar{U}$. We draw the canonical edges $\bar{U}$ and $\bar{D}$ so that they both perpendicularly cross the NSEW plane $\mathcal{P}$ containing the origin, and so that $\tau$ lies above $\mathcal{P}$, and $\beta$ lies below it. To set the lengths of $\bar{U}$ and $\bar{D}$, we subdivide $\bar{U}$ and $\bar{D}$ at the points of intersection with $\mathcal{P}$. Let $u_{1}$ denote the length to be assigned to the first portion of $\bar{U}$, below $\mathcal{P}$, and let $u_{2}$ denote the length to be assigned to the second portion of $\bar{U}$, above $\mathcal{P}$ (i.e. the length of $\bar{U}$ is $u_{1}+u_{2}$ ). Continuing around the cycle, let $d_{1}$ denote the length of the first portion of $\bar{D}$, above $\mathcal{P}$, and let $d_{2}$ denote the length of the second portion of $\bar{D}$, below $\mathcal{P}$. 
First we ensure the closure of the cycle $\Gamma(\sigma)$. To do this, we require that the sum of the lengths assigned to $U$ edges equals the sum of the lengths assigned to $D$ edges, both above and below the plane $\mathcal{P}$. For any subsequence $\gamma$ of $\sigma$, let $\Sigma_{U}(\gamma)$ denote the sum of the lengths of the edges in $\gamma$ that have direction label $U$. We will only use this notation in situations where $\gamma$ does not include the canonical edge $\bar{U}$, whose length is not yet determined. Similarly, let $\Sigma_{D}(\gamma)$ denote the sum of the lengths of the edges in $\gamma$ that have direction label $D$. Then we write the following system of equations:

$$
\begin{aligned}
& u_{2}+\Sigma_{U}(\tau)=d_{1}+\Sigma_{D}(\tau), \\
& u_{1}+\Sigma_{U}(\beta)=d_{2}+\Sigma_{D}(\beta) .
\end{aligned}
$$

Next we ensure that $\bar{U}$ is long enough that every point of $\tau$ lies above $\mathcal{P}$. Similarly, we ensure that $\bar{D}$ is long enough that every point of $\beta$ lies below $\mathcal{P}$. These conditions are captured by the following equations:

$$
\begin{aligned}
& u_{2}=1+\max \left\{\Sigma_{D}(\gamma)-\Sigma_{U}(\gamma): \gamma \text { a prefix of } \tau\right\}, \\
& d_{2}=1+\max \left\{\Sigma_{U}(\gamma)-\Sigma_{D}(\gamma): \gamma \text { a prefix of } \beta\right\} .
\end{aligned}
$$

Note that the max in each equation is at least 0 due to the empty prefix. The system of (1) and (2), which clearly has a solution, determines the lengths assigned to $\bar{U}$ and $\bar{D}$.

It remains to find the actual coordinates of the vertices in $\Gamma(\sigma)$. We can assign $u_{2}$ as the $U D$ coordinate for the vertex at the tip of $\bar{U}$, and $-u_{1}$ as the $U D$ coordinate for the vertex at the tail of $\bar{U}$. Then the $U D$ coordinates for the remaining vertices can be determined by the lengths assigned to the $U$ and $D$ edges.

The $N S$ and $E W$ coordinates of the vertices in $\Gamma(\sigma)$ can be computed in a similar fashion, after determining the lengths of $\bar{N}, \bar{S}, \bar{E}$, and $\bar{W}$.

Lemma 17 The system of equations for all six canonical edges ensures that the connectors of $\Gamma(\sigma)$ lie in distinct octants.

Proof It suffices to show that in the drawing $\Gamma(\sigma)$, any two connector paths are separated by at least one of the isothetic planes through the origin. Let $\gamma$ and $\kappa$ be two connector paths. Because we have a proper full canonical sequence, there must be at least one pair of oppositely directed canonical edges that separate the connector paths. To ease notation, suppose that the canonical edges that separate $\gamma$ and $\kappa$ are $\bar{U}$ and $\bar{D}$, and suppose furthermore that $\gamma$ lies in the path $\tau$ from $\bar{U}$ to $\bar{D}$, and $\kappa$ lies in the path $\beta$ from $\bar{D}$ to $\bar{U}$. By the system of equalities given by (1) and (2), $\bar{U}$ and $\bar{D}$ are positioned so that the $N S E W$ plane $\mathcal{P}$ through the origin separates $\tau$ from $\beta$ in the drawing. Therefore $\mathcal{P}$ separates $\gamma$ from $\kappa$ in $\Gamma(\sigma)$.

\subsubsection{Correctness of the Construction}

We are now ready to wrap up the work of Sect. 5.2. 
Lemma 18 Let $\sigma$ be a $3 D$ shape cycle of length $n$ with a proper full canonical sequence $\tau$. Then $\sigma$ is simple. Furthermore, there is an $O(n)$ time algorithm for constructing a simple drawing of $\sigma$.

Proof The algorithm has been explained in the previous two subsections. It remains to observe the following. By the simplicity of each connector drawing, no two non-canonical edges within the same connector may intersect. Furthermore, by Lemma 17, each connector lies in a distinct octant. This implies that no two noncanonical edges from distinct connectors may intersect. Finally, because each connector lies in a distinct octant, no two canonical edges may intersect. So $\Gamma(\sigma)$ is simple.

Combining Lemmas 15 and 18 gives the following result, which is the second half of our main result, Theorem 1.

Theorem 5 (Sufficiency) Every 3D shape cycle containing a full canonical sequence is simple.

\section{Algorithm}

In this section we give linear-time algorithms to test if a shape cycle is simple and to construct a simple drawing if one exists. Recall that Lemma 18 gave a linear-time algorithm to construct a simple drawing of a shape cycle from a proper full canonical sequence.

We begin with an algorithm to find a proper full canonical sequence in a shape cycle if one exists:

Lemma 19 Let $\sigma$ be a $3 D$ shape cycle of length $n$. There is an $O(n)$-time algorithm for testing whether $\sigma$ has a proper full canonical sequence, and finding one if so.

Proof The idea is to try all of the finitely many proper full canonical sequences $\tau$, and search for $\tau$ in $\sigma$ using a greedy linear scan of $\sigma$. The only complication is that elements of $\tau$ that lie in the same flat of $\sigma$ must be consecutive.

Begin by breaking $\sigma$ (arbitrarily) to form a path $\sigma^{\prime}$. Create all the 6 ! permutations of $\{U, D, N, S, E, W\}$ and discard those that have oppositely directed pairs that are adjacent in cyclic order. Let $\tau$ be such a sequence. For example, $\tau=N D E S W U$. Then for each pair of consecutive elements of $\tau$ (wrapping around the cycle), we choose whether they should share a flat or not. There are $2^{6}$ possibilities. Let $\tau^{\prime}$ be a resulting sequence. For notation, we use a dash to indicate a change of flat. For example, $\tau_{1}^{\prime}=N-D E S-W-U$ - has $D E$ sharing a flat, $E S$ sharing a flat, and the other elements in distinct flats, and $\tau_{2}^{\prime}=N D-E S W-U$ has $E S W$ in one flat and $U N D$ in another. A sequence of elements in $\tau^{\prime}$ between two dashes is called a block. For example, $D E S$ is a block of $\tau_{1}^{\prime}$. Let $m$ be the number of blocks of $\tau^{\prime}$.

Now we search $\sigma^{\prime}$ for $\tau^{\prime}$, beginning at the start of $\sigma^{\prime}$ and the start of $\tau^{\prime}$ and enforcing the properties that: (1) each block of $\tau^{\prime}$ appears consecutively in $\sigma^{\prime}$; and (2) the last element of block $i$ and the first element of block $i+1$ do not lie in a common flat of $\sigma^{\prime}$. 
Suppose first that $\tau^{\prime}$ ends in a dash, i.e. that the first and last elements of $\tau^{\prime}$ are not in a common block. For $i=1, \ldots, m$, scan forward in $\sigma^{\prime}$ to find the first occurrence of block $i$; then scan forward in $\sigma^{\prime}$ to the first element that does not lie in a common flat with the last matched element.

If this algorithm succeeds in matching all the blocks of $\tau^{\prime}$ into $\sigma^{\prime}$, then we do a final check of $\sigma$ to ensure that the last element of block $m$ does not share a flat with the first element of block 1 . Note that there is no need to check if four elements of $\tau^{\prime}$ share a flat, because the remaining two elements of $\tau^{\prime}$ would form an adjacent oppositely directed pair, which we ruled out.

In case $\tau^{\prime}$ does not end with a dash, block 1 contains the first element and the last element of $\tau^{\prime}$, and we must do some extra work to handle this "wrap-around" block. The first element of $\tau^{\prime}$ must match the first element of $\sigma^{\prime}$; the last element of $\tau^{\prime}$ must match the last element of $\sigma^{\prime}$; and if block 1 has other elements, then they must be consecutive in $\sigma^{\prime}$ (at the start or end of $\sigma^{\prime}$ as appropriate). After matching block 1, set $i=2$, scan forward in $\sigma^{\prime}$ to the first element that does not lie in a common flat with the last matched element, and proceed with the general loop above, and the final check between block $m$ and block 1 .

Correctness is justified by the observation that if there is some match $M$ of $\tau^{\prime}$ into $\sigma^{\prime}$, then $M$ must match a wrap-around block as described above, and after that we can modify $M$ by moving each successive block of $\tau^{\prime}$ earlier in $\sigma^{\prime}$ to correspond to the choice made in the algorithm. The algorithm takes linear time because there are finitely many choices for $\tau^{\prime}$, and for each such $\tau^{\prime}$, the scan takes linear time.

We now conclude with our main algorithmic result:

Theorem 6 Let $\sigma$ be a $3 D$ shape cycle of length $n$. There is an $O(n)$-time algorithm for testing whether $\sigma$ is simple, and if it is, for constructing a simple drawing of $\sigma$.

Proof We first claim that a $3 D$ shape cycle $\sigma$ is simple if and only if it contains a proper full canonical sequence:

\begin{tabular}{|c|c|c|}
\hline & & $\sigma$ has a full canonical sequence \\
\hline by & $\longrightarrow$ & $\sigma$ is simple \\
\hline & $\Rightarrow$ & $\sigma$ has a full canonical sequence \\
\hline
\end{tabular}

By this chain of implications, all these properties are equivalent. The algorithm is based on the property of having a proper full canonical sequence. Lemma 19 gave an algorithm to test if a shape cycle has a proper full canonical sequence, and to find such a sequence when possible. Lemma 18 gave a linear-time algorithm to construct a simple drawing of a shape cycle from a proper full canonical sequence.

\section{Conclusions and Open Problems}

This paper has characterized simple shape cycles, that is, shape cycles that admit a simple orthogonal drawing in $3 D$, i.e. a drawing such that no two edges intersect 
except at common endpoints. The characterization yields linear-time recognition and drawing algorithms. It provides an extension to $3 D$ space of the well-known characterization of simple shape cycles in $2 D$ by Vijayan and Widgerson [10].

We make one remark about the volume required for a simple drawing of a shape cycle with vertices at grid points. Our construction draws a shape cycle of $n$ edges within an $n \times n \times n$ bounding box. This is because the connectors are drawn using an expanding drawing, so each new edge adds 1 to its axis of the bounding box. Also, each of the six canonical edges is drawn as short as possible and adds 1 to its axis of the bounding box. ${ }^{1}$ There is a matching lower bound of $\Omega\left(n^{3}\right)$ : a sequence that repeats $E S W N n$ times forms a spiral that requires width at least $2 n$ in the $E W$ and $N S$ axes, and adding a spiral that repeats $U N D S n$ times requires width $2 n$ in the $U D$ axis as well. Therefore the worst-case volume required to draw a shape cycle of $n$ edges is $\Theta\left(n^{3}\right)$.

There are a number of natural open questions arising from our work:

1. Is the following problem NP-hard? Given a simple shape cycle, minimize the volume of a simple drawing with vertices at grid points.

2. Characterize simple shapes for graphs that are not just cycles. Some preliminary results in this direction can be found in [6].

3. Extend the characterization of this paper to simple shape cycles with more than six directions and/or to dimension higher than three.

4. Is it true that shape cycles in $3 D$ are more likely to be simple than those in $2 D$ ? What is the probability that a random shape cycle in $3 D$ is simple?

5. The final problems concern shape knots. Which simple shape cycles can be drawn as the trivial knot (with no two edges intersecting except at common endpoints)? Which knots can be created from a given simple shape cycle?

Acknowledgements We thank an anonymous referee for careful reading and for the result about volume. Research partially supported by operating grants from the Natural Sciences and Engineering Research Council (NSERC) of Canada, and by MIUR of Italy under project AlgoDEEP prot. 2008TFBWL4.

\section{References}

1. Di Battista, G., Vismara, L.: Angles of planar triangular graphs. SIAM J. Discrete Math. 9(3), 349359 (1996)

2. Di Battista, G., Eades, P., Tamassia, R., Tollis, I.: Graph Drawing. Prentice Hall, New York (1999)

3. Di Battista, G., Liotta, G., Lubiw, A., Whitesides, S.: Orthogonal drawings of cycles in 3D space (extended abstract). In: Marks, J. (ed.) Graph Drawing, Proc. GD '00. LNCS, vol. 1984, pp. 272-283. Springer, Berlin (2001)

4. Di Battista, G., Liotta, G., Lubiw, A., Whitesides, S.: Embedding problems for paths with direction constrained edges. Theor. Comput. Sci. 289(2), 897-917 (2002)

5. Garg, A.: New results on drawing angle graphs. Comput. Geom. Theory Appl. 9(1-2), 43-82 (1998). Special Issue on Geometric Representations of Graphs, G. Di Battista and R. Tamassia, eds.

6. Giacomo, E.D., Liotta, G., Patrignani, M.: A note on 3D orthogonal drawings with direction constrained edges. Inf. Process. Lett. 90(2), 97-101 (2004)

\footnotetext{
${ }^{1}$ Strictly speaking, the canonical edges add 2 units of length, as our construction does not place any vertices on isothetic planes through the origin, but it is easy to compress the empty planes away.
} 
7. Patrignani, M.: Complexity results for three-dimensional orthogonal graph drawing. J. Discrete Algorithms 6(1), 140-161 (2008)

8. Tamassia, R.: On embedding a graph in the grid with the minimum number of bends. SIAM J. Comput. 16(3), 421-444 (1987)

9. Tamassia, R.: Handbook of Graph Drawing and Visualization. Chapman \& Hall/CRC, New York (2009). In press

10. Vijayan, G., Wigderson, A.: Rectilinear graphs and their embeddings. SIAM J. Comput. 14, 355-372 (1985)

11. Vijayan, V.: Geometry of planar graphs with angles. In: Proc. 2nd Annu. ACM Sympos. Comput. Geom, pp. 116-124 (1986) 